\title{
Whole-exome Sequencing Identify Rare Variants in Novel Candidate Genes with Non-syndromic Patent Ductus Arteriosus
}

\section{Ying Gao}

Shidong Hospital

\section{Ying Liu}

Shidong Hospital

Jiaoyu Li

Xin Hua Hospital, School of Medicine, Shanghai Jiao Tong University

\section{Yinghui Chen}

Xin Hua Hospital, School of Medicine, Shanghai Jiao Tong University

\section{Qi Zhang}

Xin Hua Hospital, School of Medicine, Shanghai Jiao Tong University

\section{Bingyao Zhang}

Xin Hua Hospital, School of Medicine, Shanghai Jiao Tong University

\section{Pengjun Zhao}

Xin Hua Hospital, School of Medicine, Shanghai Jiao Tong University

\section{Bo Chen ( $\nabla 1205293877 @ q q . c o m$ )}

Xin Hua Hospital, School of Medicine, Shanghai Jiao Tong University

\section{Research Article}

Keywords: Congenital heart defects, Patent Ductus Arteriosus, Whole-exome sequencing, Rare variants

Posted Date: January 15th, 2021

DOI: https://doi.org/10.21203/rs.3.rs-142511/v1

License: (1) (1) This work is licensed under a Creative Commons Attribution 4.0 International License. Read Full License 
1 Whole-exome Sequencing Identify Rare Variants in Novel Candidate

2 Genes with Non-syndromic Patent Ductus Arteriosus

3 Ying Gao ${ }^{1 \#}$, Ying Liu ${ }^{1 \#}$, Jiaoyu Li ${ }^{2 \#}$, YingHui Chen ${ }^{2}$, Qi Zhang ${ }^{2}$, Bingyao Zhang ${ }^{2}$, Pengjun $Z_{\text {hao }}^{2 *} \&$

4 Bo $\mathrm{Chen}^{2 *}$

$5 \quad{ }^{1}$ Department of Pediatric, Shidong Hospital, Shanghai, China

$6 \quad{ }^{2}$ Department of Pediatric Cardiology, Xin Hua Hospital, School of Medicine, Shanghai Jiao Tong

7 University, Shanghai, China

$8 \quad{ }^{*}$ Correspondence: Pengjun Zhao: pjunzhao@sina.com; Bo Chen: 1205293877@qq.com

$9 \quad$ "Ying Gao, Ying Liu and Jiaoyu Li contribute to this study equally. 


\section{Abstract}

17 Background

18 Patent Ductus Arteriosus (PDA) is one of the most common congenital heart defects that can cause

19 pulmonary hypertension, heart failure, and even death. Prior studies have suggested a role for genetics

20 in determining spontaneous ductal closure, however the clinical characteristics and genetic cause

21 underlying PDA remain unclear.

\section{Results}

23 Therefore, to further explore genetic etiology of PDA, we applied Whole-exome Sequencing (WES) in

2439 unrelated isolated, non-syndromic PDA patients and 100 healthy controls. Through a series of bio-

25 information filtering strategies, the candidate genes are prioritized by comprehensively considering

26 factors such as gene functional enrichment, expression pattern and mutation burden during heart

27 development. 18 rare damage variants of 6 total novel genes (SOX8, NES, CDH2, ANK3, EIF4G1, HIPK1)

28 were identified for the first time and these pathogenic candidates are also highly expressed in the heart

29 of human embryos.

30 Conclusions

31 WES is an efficient diagnostic tool for identifying PDA related genes. The finding of our study 32 contributes new insights into the molecular basis of PDA and may inform further studies on genetic risk 33 factors for this congenital birth defect.

Keywords: Congenital heart defects, Patent Ductus Arteriosus, Whole-exome sequencing, Rare variants 


\section{Background}

39 The ductus arteriosus (DA) is a normal fetal structure connecting the pulmonary artery and descending

40 aorta to maintain blood circulation in fetal period[1]. It becomes pathological if it remains patent after

41 birth[1]. Failure of the ductus arteriosus to close after birth is termed patent ductus arteriosus (PDA) and is one of the most common heart defects. It is accounting for $15 \%-20 \%$ of the total number of congenital heart defect. Its incidence is about $1 / 2000$ in term infant and $8 / 1000$ in premature infant[2]. Persistent ductal shunting may lead to pulmonary overcirculation and induce systemic hypoperfusion, increasing the risk of pulmonary hypertension, infective endocarditis, heart failure and even death[3]. in healthy term infants[4]. This process occurs by abrupt contraction of the muscular wall of the PDA, which is associated with a balance of neurohumoral factors. The increase of contractile elements, such as PO2 and endothelin-1, and the decrease of relaxants, such as PGE2 levels and nitric oxide, are the main factors to cause the closure of ductus arteriosus[4]. Under the action of these hormones, neural-

51 crest-derived cells migrate into the subendothelial space, transform to vascular smooth muscle cells

52 (VSMCs). Then with the contraction of the medial membrane and the circular muscle in the ductus

53 arteriosus, the lumen is shortened and finally closed[5]. The occurrence of PDA has both inherited and

54 acquired causes. However, the etiology and pathogenesis are still not completely known yet.

55 The understanding of the genetic mechanism of PDA initially came from the syndrome type patent 56 ductus arteriosus. Chromosomal abnormalities, including aneuploidy and microdeletion, are the most 
59 Syndrome (47, XXY), etc. [6-8]. Other than chromosomal rearrangements, a single gene mutation can

60 also cause syndromic PDA, including Noonan Syndrome (PTPN11 mutation), Holt-Oram Syndrome

61 (TBX5 mutation) and Char Syndrome (TFAP2B mutation)[9-11]. With the fruition for human genome

62 sequencing, genetic factors assume a paramount part in the pathogenesis of PDA. However, little is

63 known about the genetic mechanism of isolated non-syndromic patent ductus arteriosus. Previous studies

64 have demonstrated that rare damaging mutations in MYH11, TFAP2B were detected in some isolated non-syndromic PDA patients[12]. Erdogan et al. conducted an array comparative genome screening in 105 patients with CHD and found a 1.92MB deletion in 1q21.1(CJA5) in an isolated PDA patient[13].

67 However, most of previous studies focus on the known pathogenic gene mutations of syndromic PDA.

68 The molecular genetic mechanisms of non-syndromic PDA are still largely unknown. Using WES and

69 bioinformatics methods to detect rare variants associated to PDA have never been reported yet.

70 In our study, to systematically examine the clinical characteristics and genetic cause of isolated, non-

71 syndromic PDA, we recruited 39 unrelated, isolated, non-syndromic PDA patients and 100 healthy 72 children to performed whole exome sequencing (WES). Through a series of bioinformatics filtering steps,

73 we identified 18 rare damaging variants in 6 candidate genes (SOX8, NES, CDH2, ANK3, EIF4G1,

74 HIPK1). Notably, we found that these candidate genes are highly expressed in human embryonic hearts.

75 Therefore, we hope our discovery of the pathogenic genes could fill the underlying mechanism of PDA

76 and promote further experimental analysis.

\section{Methods}


81 were assessed by echocardiography or cardiac catheterization in both groups. And the case groups were and International Ethical Guidelines for Health-related Research Involving Humans.

DNA extraction and Whole Exome Sequencing

89 The genomic DNA of all participants was extracted from blood samples by using the QIAamp DNA Blood Mini Kit (QIAGEN, Germany). DNA samples were stored at $-80^{\circ} \mathrm{C}$ until further use. Genomic DNA was eluted, purified, and amplified by ligation-mediated PCR and then subjected to DNA sequencing on the Illumina platform. Qualified DNA samples from the groups of PDA and controls were performed WES to detecting rare variations. The Clean data was obtained by removing adaptor

94 sequences and low- quality reads.

\section{SNP identified and Quality fliting}


101 joint genotyping. Variant quality control and filtering were performed based on variant quality score

102 recalibration (VQSR) by building GMM model[16]. We used ANNOVAR53 to annotate the variants for

103 functional and population frequency information. All potentially damaging variants on the candidate

104 genes were classified into five groups, including pathogenic, likely pathogenic, variant uncertain

105 significance, likely benign and benign[17]. We fileted for rare damaging variants with the following

106 criteria: (1) read quality $>20 \mathrm{bp}$, (2) minor allele frequency (MAF) $<5 \%$ (3) variants frequency $<1 \%$ in

107 1000G database, ESP6500 database, Exac database and gnomAD database, (4) Removing small (<10bp)

108 non-shift indel mutation in Repeat region, (5) fliting out synonymous mutations and non-synonymous

109 mutations that are not predicted to be deleterious by PolyPhen, SIFT, or MutationTaster tools, (6) fliting

110 out the variants without annotation information in all exome database[17].

\section{Variants Fliting based on Fisher Exact test and Burden analysis}

112 Differences in baseline characteristics between cases and controls were assessed by a Fisher exact test

113 for categorical variables with the "R" statistical package. For comparisons, $\boldsymbol{P}$-value $<0.05$ was

114 considered statistically significant. Subsequently, for capturing rare target genes in a limited range, we

115 aggregated the SNP data based on the gene level and conducted gene-based Burden analysis to increase

116 statistical power. The different variant sites located in the same gene were put together as a whole for

117 disease association analysis. We filtered for candidate genes based on Burden analysis with the following

118 criteria: (1) $\boldsymbol{P}$-value $<0.05$ or FDR $<0.05$, (2) hit by at least one variant in 3 cases (3) not found in any

119 sample of control group. Then we prioritized genes based on Fisher exact test and Burden analysis.

\section{Functional enrichment analysis and Network analysis}


121 To further fliting the candidate gene associated with PDA, we performed functional enrichment

122 analysis to identify the function of above candidate genes. Pathway analysis of the candidate gene

123 profiling results was performed using the Gene ontology (GO version: 30.10 .2017$)$ and KEGG pathway

124 (http://www.genome.jp/kegg/pathway.html) mapping within the web-based tool database for annotation,

125 visualization and integrated discovery[18, 19]. The significant threshold was set to be an adjusted P-

126 value $<0.05$. In addition, we also prioritized those genes based on functional enrichment analysis.

127 Furthermore, to detect relationship between our candidate genes and known disease-causing genes, we

128 performed a protein-protein interaction (PPI) network analysis[20]. PPI gene network was generated by

129 Cytoscape software based on STRING database.

130 Tissue collection and Expression detection

131 In addition to the genes prioritized above, we also prioritized genes according to the expression in

132 human embryonic heart. Previous studies have divided eight embryonic weeks (56 days) into 23

133 internationally accepted Carnegie stages[21]. To further investigate the potential function of our

134 candidate gene, we collected human embryonic heart in different Carnegie stages from S10 to S16 after

135 medical termination of pregnancy from Xinhua hospital. RNA was extracted and purified by Experion

136 automated gel electrophoresis system and e RNeasy MinElute Cleanup Kit. Then we used Affymetrix

137 HTA 2.0 microarray to detect the expression patterns of our candidate genes.

\section{$138 \quad$ Results}


Table 1: Characteristics of 39 PDA Patients

\begin{tabular}{|c|c|}
\hline Patients Characteristics & Numbers \\
\hline Age & $2.92 \pm 2.44$ \\
\hline Male n $(\%)$ & $15(38 \%)$ \\
\hline Female n $(\%)$ & $24(61 \%)$ \\
\hline Male-to-Female ration $\quad(\%)$ & $62 \%$ \\
\hline BMI $(\mathrm{kg} / \mathrm{m} 2)$ & $16.58 \pm 4.34$ \\
\hline PDA size $(\mathrm{mm})$ & $2.87 \pm 1.68$ \\
\hline Birth weight（kg） & $2.96 \pm 0.73$ \\
\hline Gestational age（week） & $39.04 \pm 1.46$ \\
\hline \multicolumn{2}{|l|}{ Associated cardiac defect n (\%) } \\
\hline VSD n $(\%)$ & $2(5 \%)$ \\
\hline ASD n $(\%)$ & $7(18 \%)$ \\
\hline Others n $(\%)$ & $2(5 \%)$ \\
\hline
\end{tabular}

All values are expressed as mean $\pm \mathrm{SD}$ or $\mathrm{n}(\%)$.

\section{Population}

14039 unrelated isolated, non-syndromic PDA patients (Han Chinese) and 100 healthy children were

141 recruited in Xinhua hospital with ages ranging from 2 months to 13 years. Among these patients, $28 \%$ 
143 septal defect (VSD, $\mathrm{n}=2$ ) and others $(\mathrm{n}=2)$ (Table 1$)$. All our samples are full-term, and no one was

144 accompanied by other major cardiac structural abnormalities or developmental syndrome. Whole-exome

145 sequencing, performed in all samples at an average depth of coverage of approximately 105 times per

146 base, identified 411344 single-nucleotide variants (SNVs) and 23101 indels across the genome. Through

147 a series of filtering strategies mentioned in Figure 1, 11910 rare damaging variants were screen with a

148 threshold of minor allele frequency (MAF) at $0.5 \%$. As illustrate in figure 2 , we found more rare

149 damaging variants in PDA group than control group, which was observed in splice site, nonsense

150 mutation and missense mutation. Consistently, the mutation type of $\mathrm{C}>\mathrm{T}$ and $\mathrm{G}>\mathrm{A}$ accounted for the

151 majority of base mutations compared with other types (Figure 2). Based on these mutations, we next

152 adopted a bioinformatic filtering strategy to identify candidate genes associated to PDA.

\section{$153 \quad$ Figure 1}

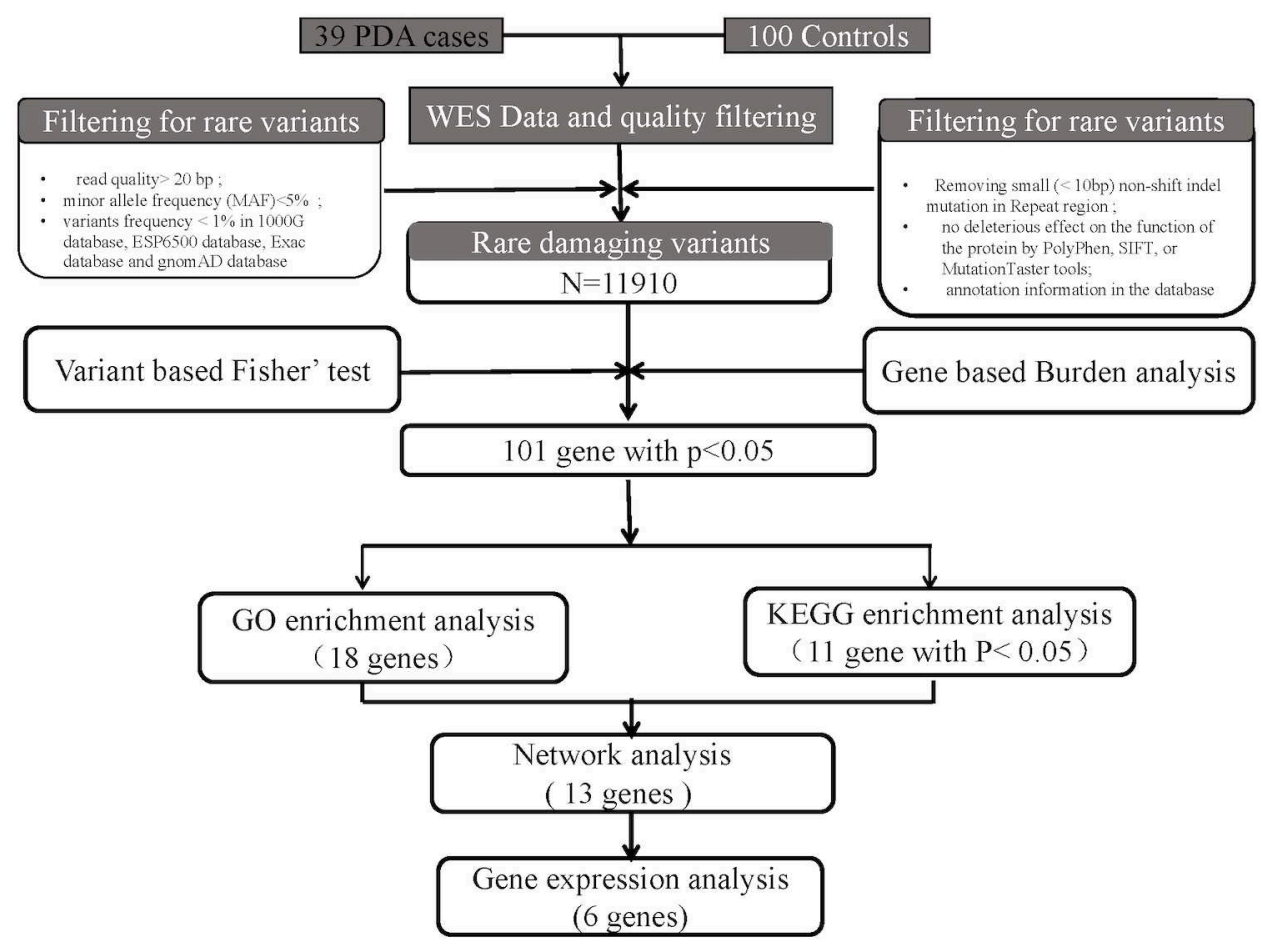

\section{$155 \quad$ Figure 2}



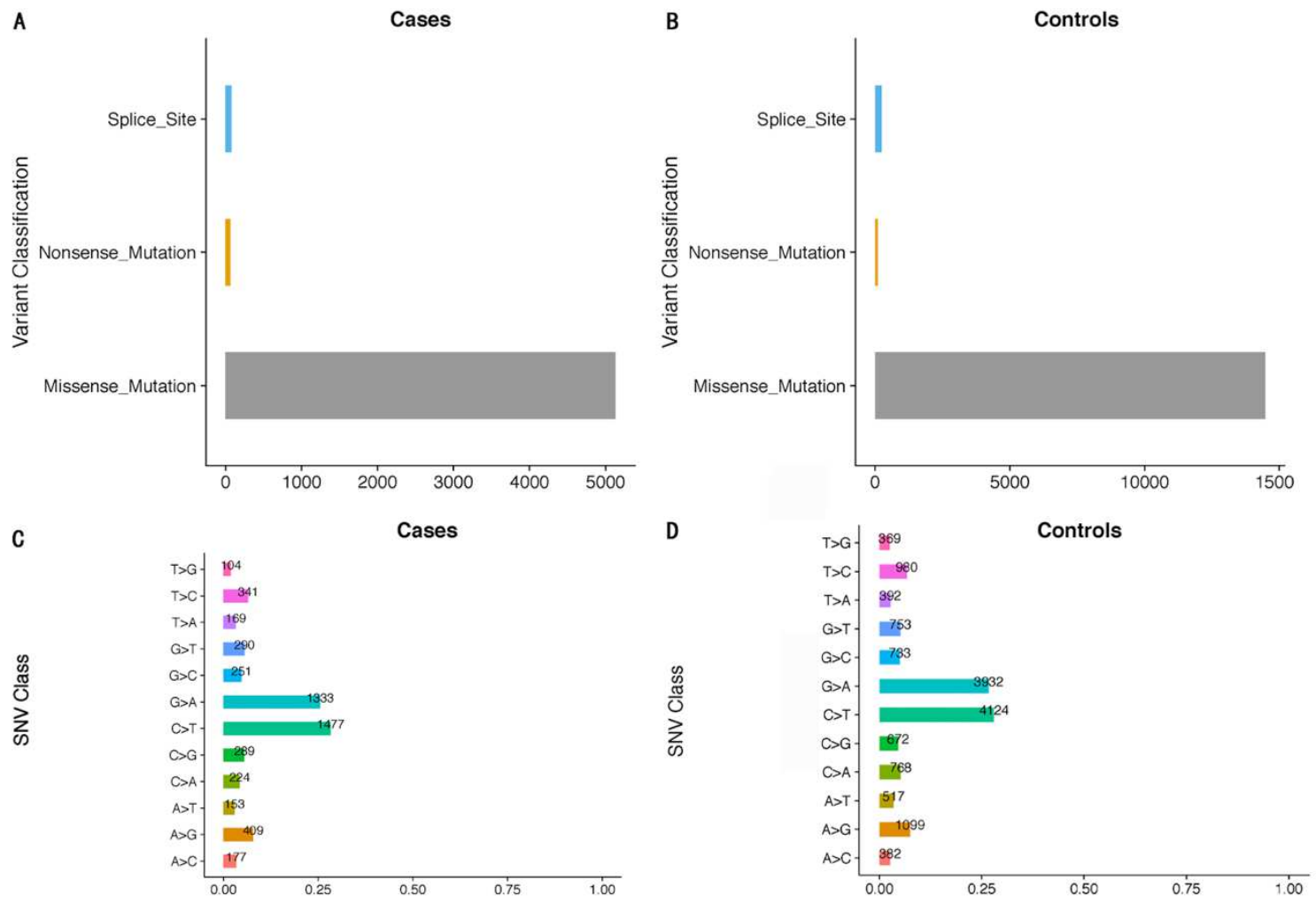

\section{$157 \quad$ Variants identified based on Fisher exact test}

158 To investigate the genetic cause of PDA, we next used Fisher exact test to the p-value of allele

159 frequency for each SNP between case and control group. Then we genotype these snps and identified 44

160 candidate snps located within genes, based on an empirical false discovery rate (FDR) of 0.05 or P-valve

1610.05 (Table 2). And we prioritized the variants based on Fisher exact test and showed the top ten snps

162 with statistically significant in Figure 3. Notably, we found that snp rs103826685 and snp rs32552095

163 located in gene SLC9B1 and HLA-DRB1 were significantly enriched $(\mathrm{P}<0.0001)$. Comparing approach

164 with the single-point analysis, carried out with Fisher exact test, we obtain that SNP rs103826685 is the most significantly associated. 


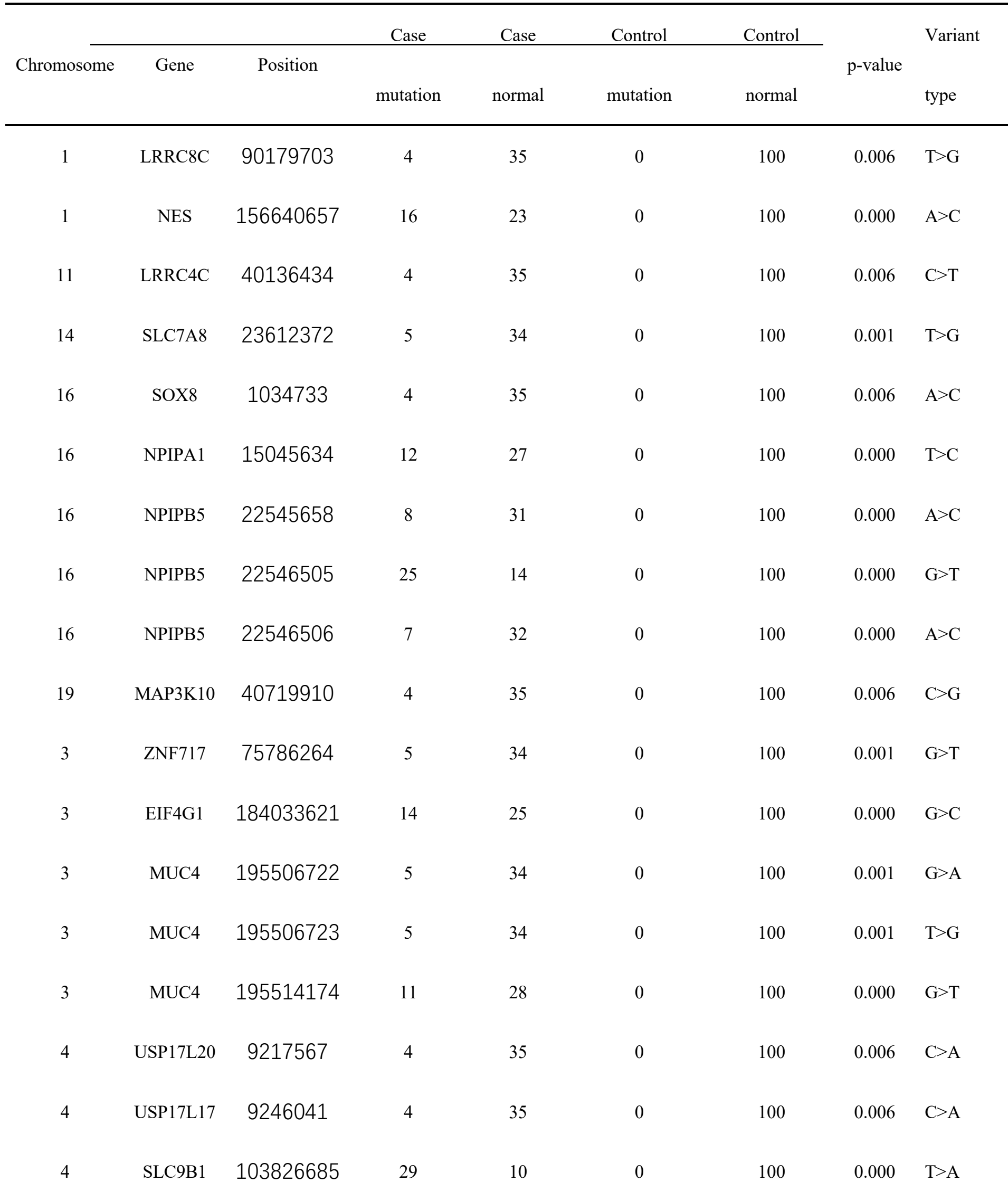


HLA-

6

$$
32487344
$$

6

33

0

100

$0.000 \quad \mathrm{~T}>\mathrm{C}$

DRB5

HLA-

6

32552095

25

14

0

100

0.000

$\mathrm{C}>\mathrm{T}$

DRB1

\section{Figure 3}

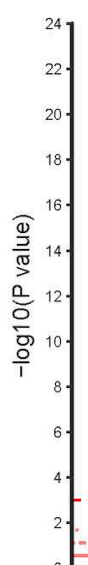

\section{Genes identified based on Burden analysis}

169 To further increase statistical power, we aggregated the SNP data at the gene level and performed

170 burden analysis. Given thresholds of 0.05 for P-value, we identified 57 genes with potential pathogenicity

171 as PDA-associated (Table 3). Subsequently, we prioritized these genes based on Burden analysis and

172 showed the top ten genes with statistically significant in Heatmap (Figure 4). Among these genes, we

173 found that NPIPB5, SLC9B1, and HLA-DRB1 were considered as the top three with high confidence.

174 Notably, SLC9B1, and HLA-DRB1 was also the most significant based on Fisher exact test. 
Table 3: Gene fliting based on Burden analysis

\begin{tabular}{|c|c|c|c|c|c|}
\hline Gene & Case mutation & Case normal & Control mutation & Control normal & $\begin{array}{c}\text { p- } \\
\text { value }\end{array}$ \\
\hline ASIC3 & 5 & 34 & 0 & 100 & 0.001 \\
\hline CFAP45 & 4 & 35 & 0 & 100 & 0.006 \\
\hline CYP21A2 & 4 & 35 & 0 & 100 & 0.006 \\
\hline EVI5 & 4 & 35 & 0 & 100 & 0.006 \\
\hline HIPK1 & 4 & 35 & 0 & 100 & 0.006 \\
\hline HLA-DRB1 & 25 & 14 & 0 & 100 & 0.000 \\
\hline HLA-DRB5 & 6 & 33 & 0 & 100 & 0.000 \\
\hline KRT39 & 5 & 34 & 0 & 100 & 0.001 \\
\hline LRRC4C & 4 & 35 & 0 & 100 & 0.006 \\
\hline MAP3K10 & 4 & 35 & 0 & 100 & 0.006 \\
\hline NPIPA1 & 13 & 26 & 0 & 100 & 0.000 \\
\hline NPIPB5 & 31 & 8 & 0 & 100 & 0.000 \\
\hline POTEE & 5 & 34 & 0 & 100 & 0.001 \\
\hline SLC9B1 & 29 & 10 & 0 & 100 & 0.000 \\
\hline SLX4 & 4 & 35 & 0 & 100 & 0.006 \\
\hline $\mathrm{SOX} 8$ & 4 & 35 & 0 & 100 & 0.006 \\
\hline TBC1D3F & 4 & 35 & 0 & 100 & 0.006 \\
\hline TCAF2 & 5 & 34 & 0 & 100 & 0.001 \\
\hline
\end{tabular}




\begin{tabular}{|c|c|c|c|c|c|}
\hline USP17L11 & 5 & 34 & 0 & 100 & 0.001 \\
\hline USP17L17 & 6 & 33 & 0 & 100 & 0.000 \\
\hline USP17L18 & 5 & 34 & 0 & 100 & 0.001 \\
\hline USP17L2 & 6 & 33 & 0 & 100 & 0.000 \\
\hline USP17L20 & 5 & 34 & 0 & 100 & 0.001 \\
\hline VARS & 4 & 35 & 0 & 100 & 0.006 \\
\hline ZNF717 & 6 & 33 & 0 & 100 & 0.000 \\
\hline
\end{tabular}

Figure 4
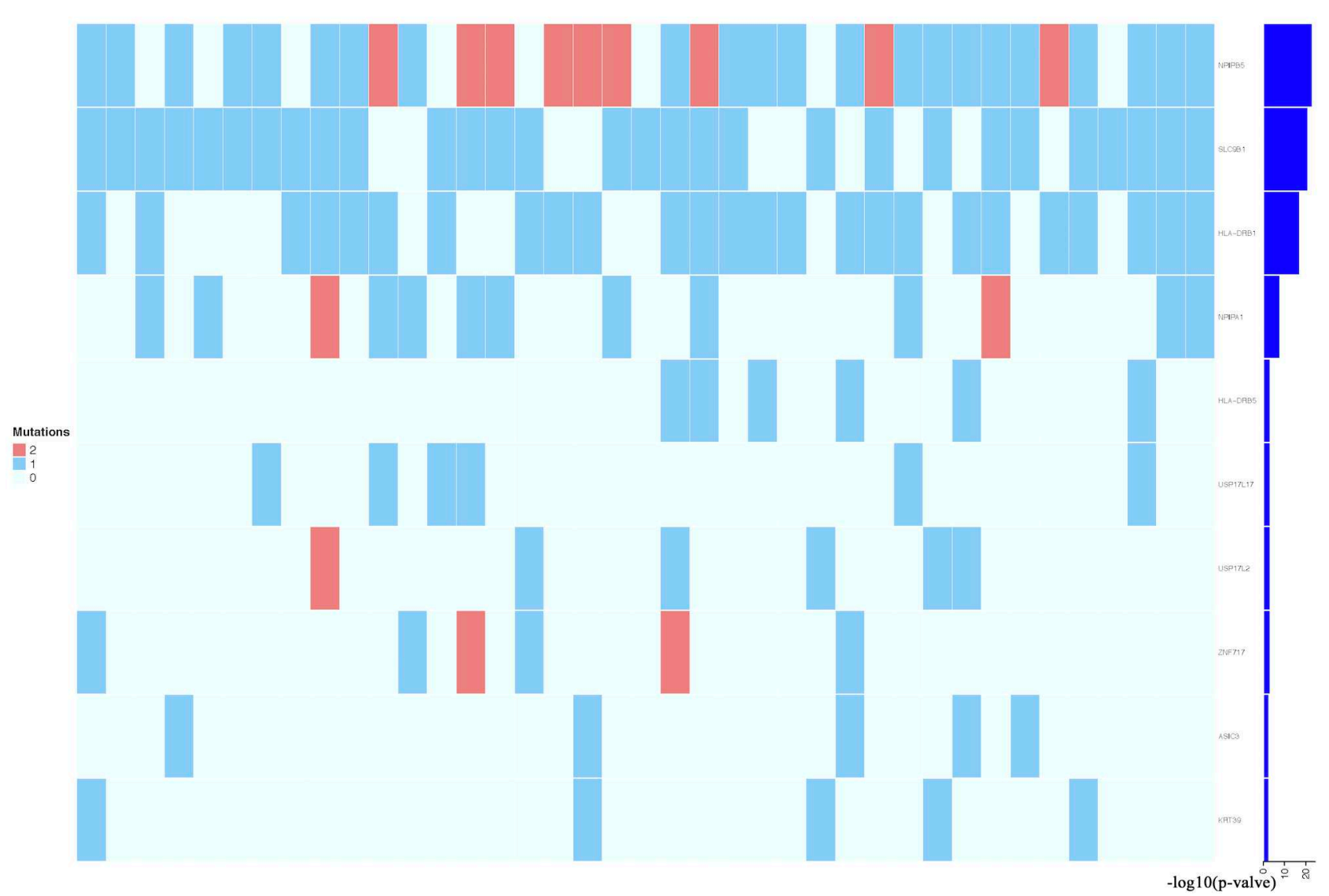

Mutations

Mutal
$\begin{array}{r}2 \\ 1 \\ 0 \\ 0\end{array}$

176

\section{Function analysis}

178 The Gene Ontology $(\mathrm{GO})$ terms $(\mathrm{p}<0.05)$ are described as a network of biological processes, which

179 are organized in a way of overlapping in space and clustered according to their relationships[18]. To 
180 further test the significance of these genes, we conducted extensive genetic functional enrichment

181 analysis. We next analyzed which Gene Ontology (GO) terms and KEGG pathways were enriched in

182 these 101 candidate genes after Fisher exact test and Burden analysis. Functional enrichment analysis of

183 differentially expressed genes revealed that Gene Ontology (GO) terms associated with thiol-dependent

184 ubiquitinyl hydrolase activity (TermID: GO:0036459), peptide antigen binding (TermID: GO:0042605)

185 and ubiquitin-dependent protein catabolic process (TermID: GO:0006511) were highly enriched in the

186 upregulated gene set. A particular focus was placed on terms representing prostaglandin, apoptosis, and

187 heart development. (Figure 5). Moreover, KEGG analysis of the direct gene targets in PDA patients

188 revealed enrichment in pathways related to Cell adhesion molecules (CAMs) (TermID: path: hsa04514,

189 pvalue:0.001), Viral myocarditis (TermID: path: hsa05416, pvalue:0.0035) and Asthma (TermID: path:

190 hsa05310, pvalue:0.01 (Figure 6). Based on the results of functional enrichment analysis, we screened

191 some pathway genes related to cardiovascular development.

$192 \quad$ Figure 5 


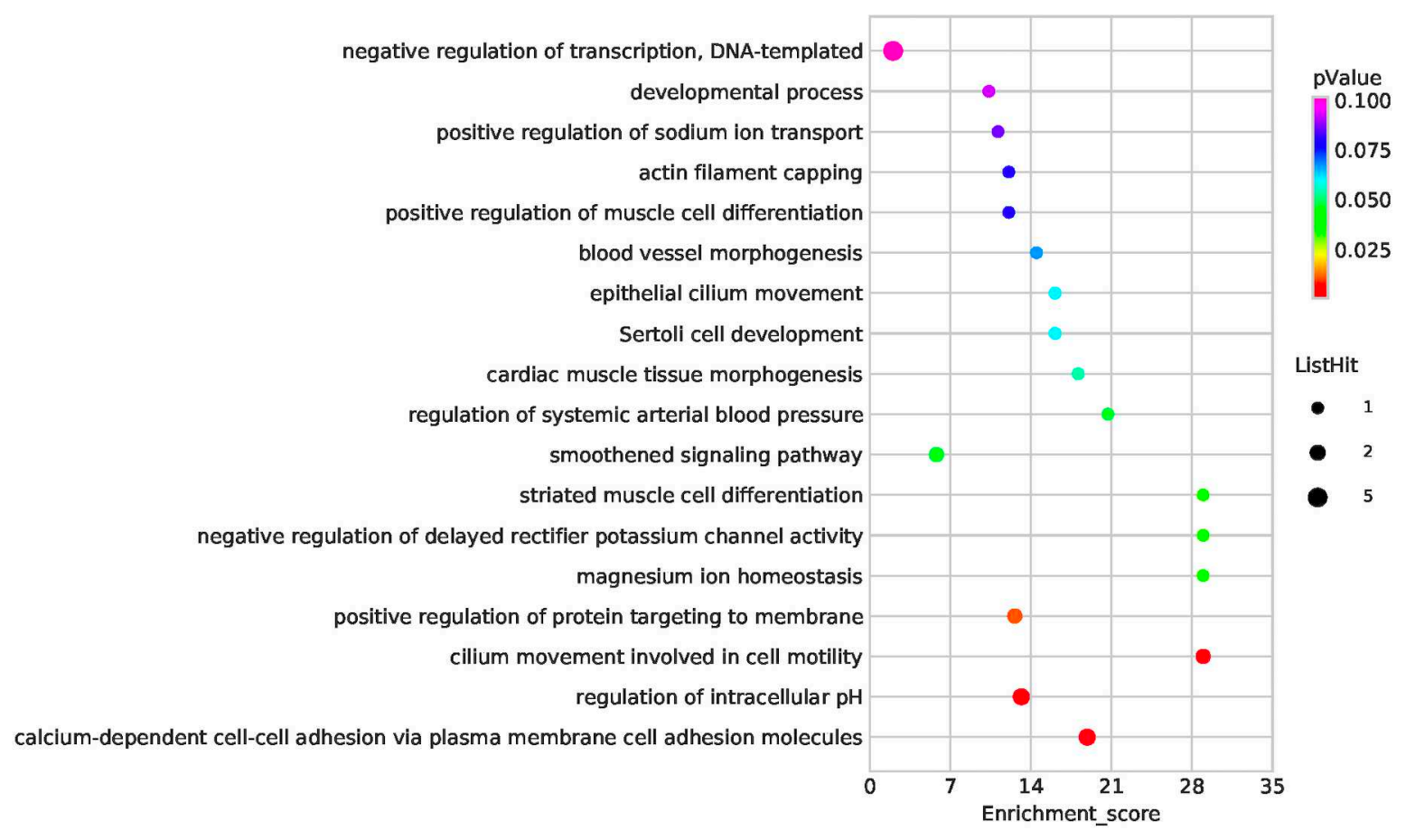

193

$194 \quad$ Figure 6 


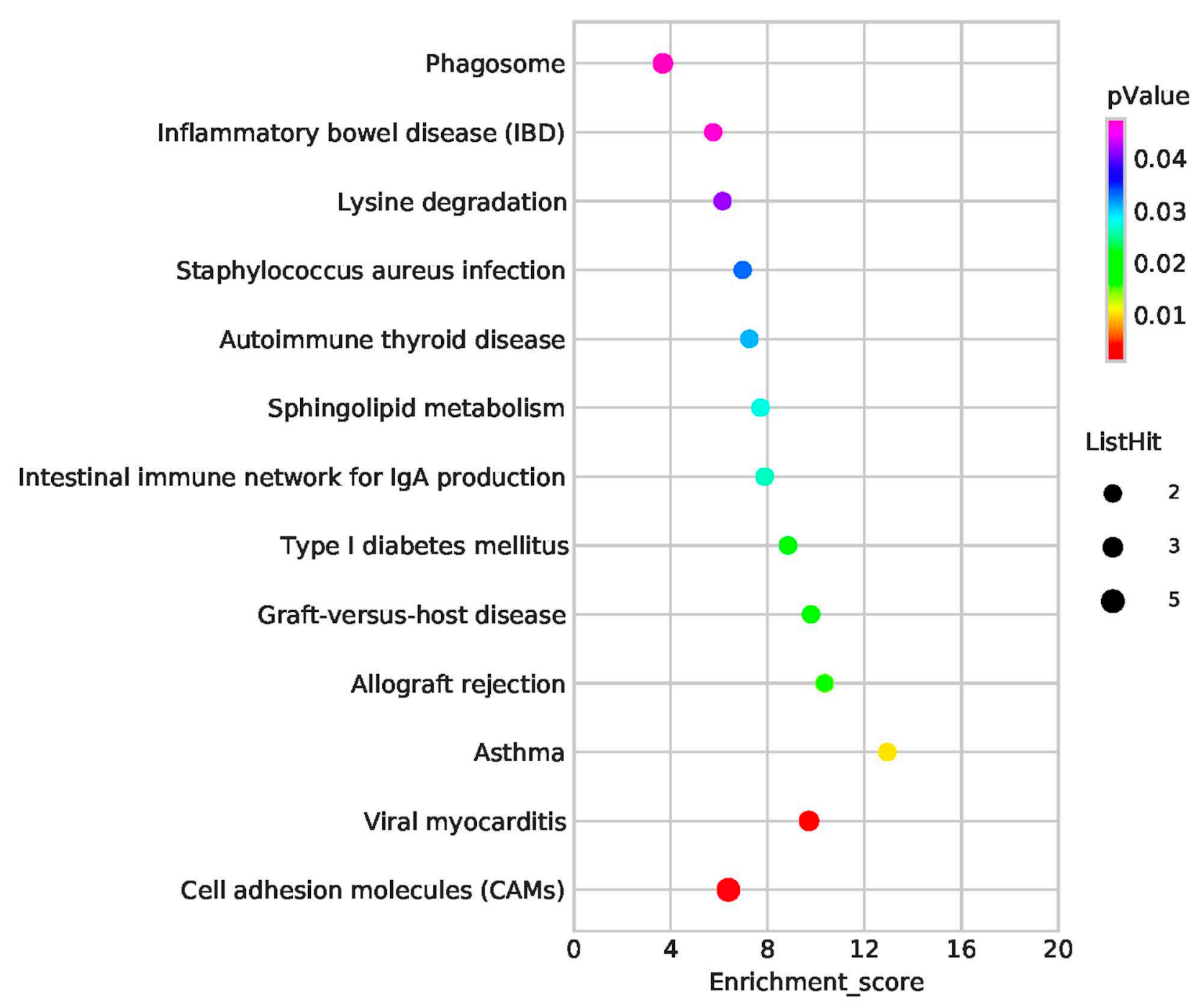

\section{Network analysis}

197 To further test the role of gene after functional enrichment analysis, we mapped the 29 our candidate

198 genes based on analysis above and 240 known pathogenic genes to the PPI network (Table S1). The

199 known genes from previous literature were divided into two different gene groups, which are related to

200 cardiac and vascular development and PDA. The result showed that NES and CDH2 had the most direct

201 and obvious relation to known pathogenic genes, both in known CHD related genes and PDA related

202 gene. Moreover, CDH2 and NES have the highest weight and located the center of PPI network (Figure 
203 7, Figure 8). Therefore, based on the degree of correlation, we screened some candidate gene for final

204 verification.

$205 \quad$ Figure 7

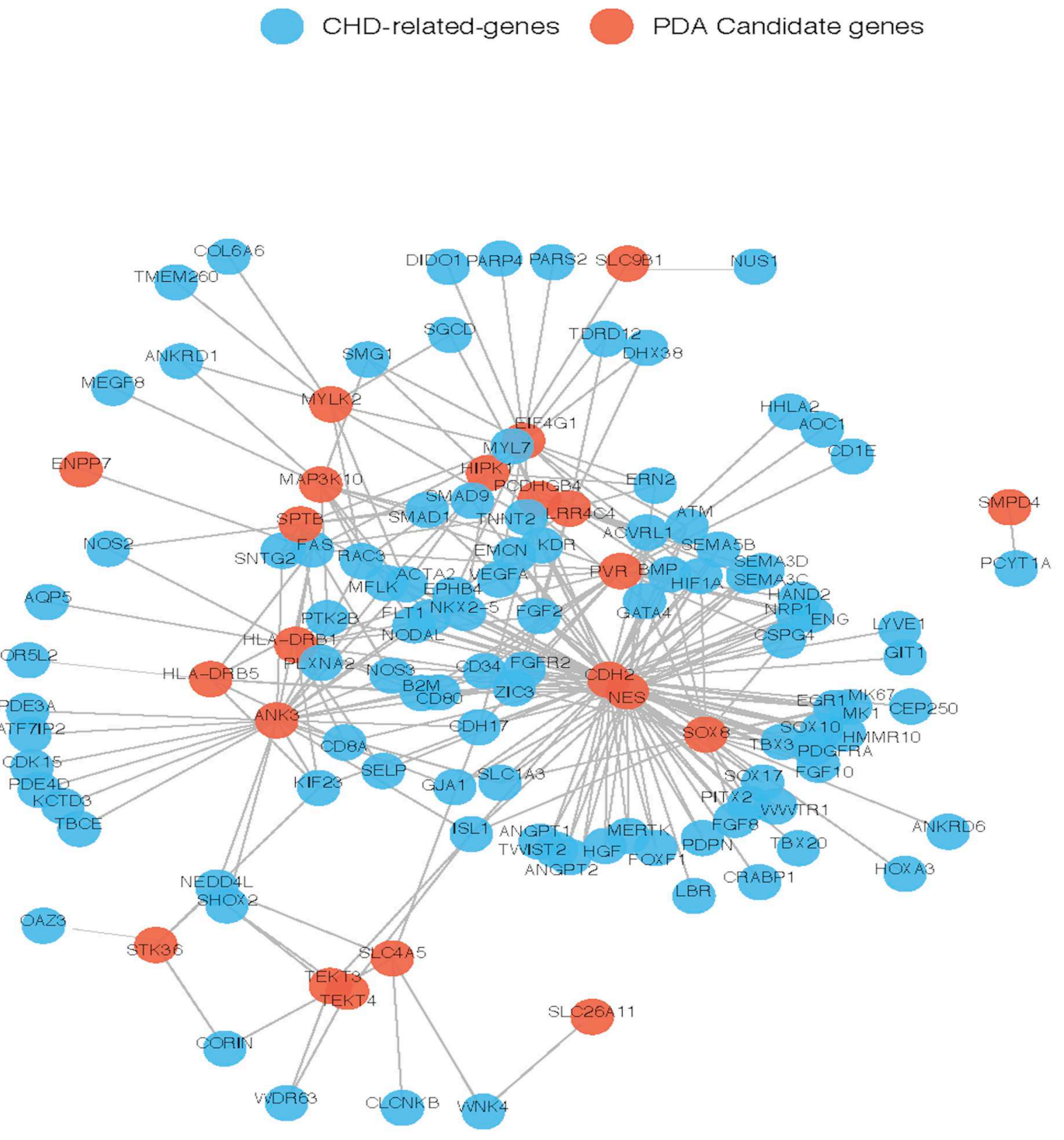

Figure 8 


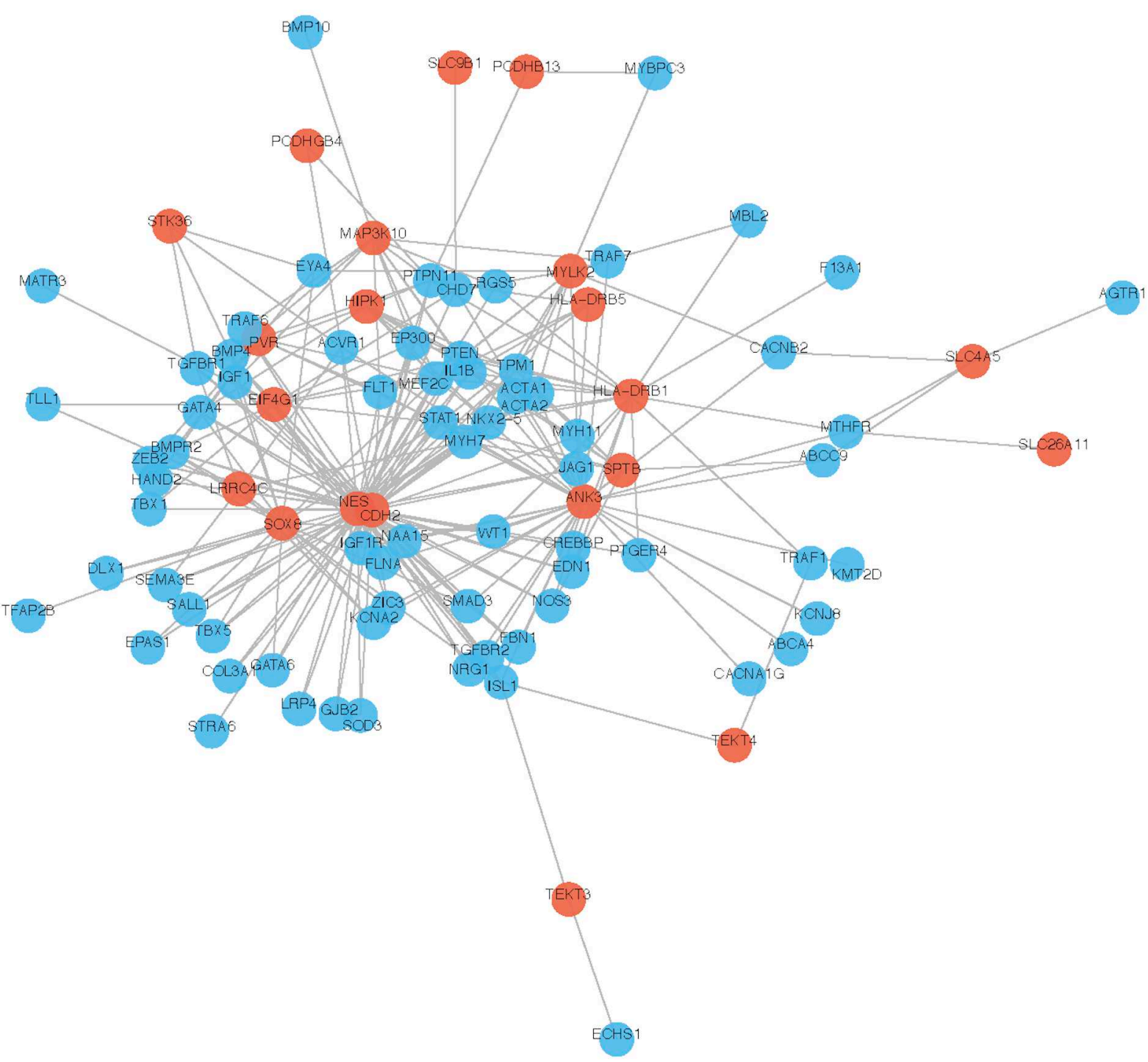

\section{Detection of candidate genes expression in human embryonic heart}

210 To further investigate the potential function of our candidate gene expression in human heart, 11 genes

211 were tested expression in human embryonic heart at different Carnegie stages. Then we prioritized those

212 candidate gene according to the amount of expression and identified final 6 pathogenic genes (SOX8, NES,

213 CDH2, ANK3, EIF4G1 and HIPK1) (Figure 9). Among them, we found that CDH2 expressed the most

214 highly in the embryonic heart (Figure 10). 

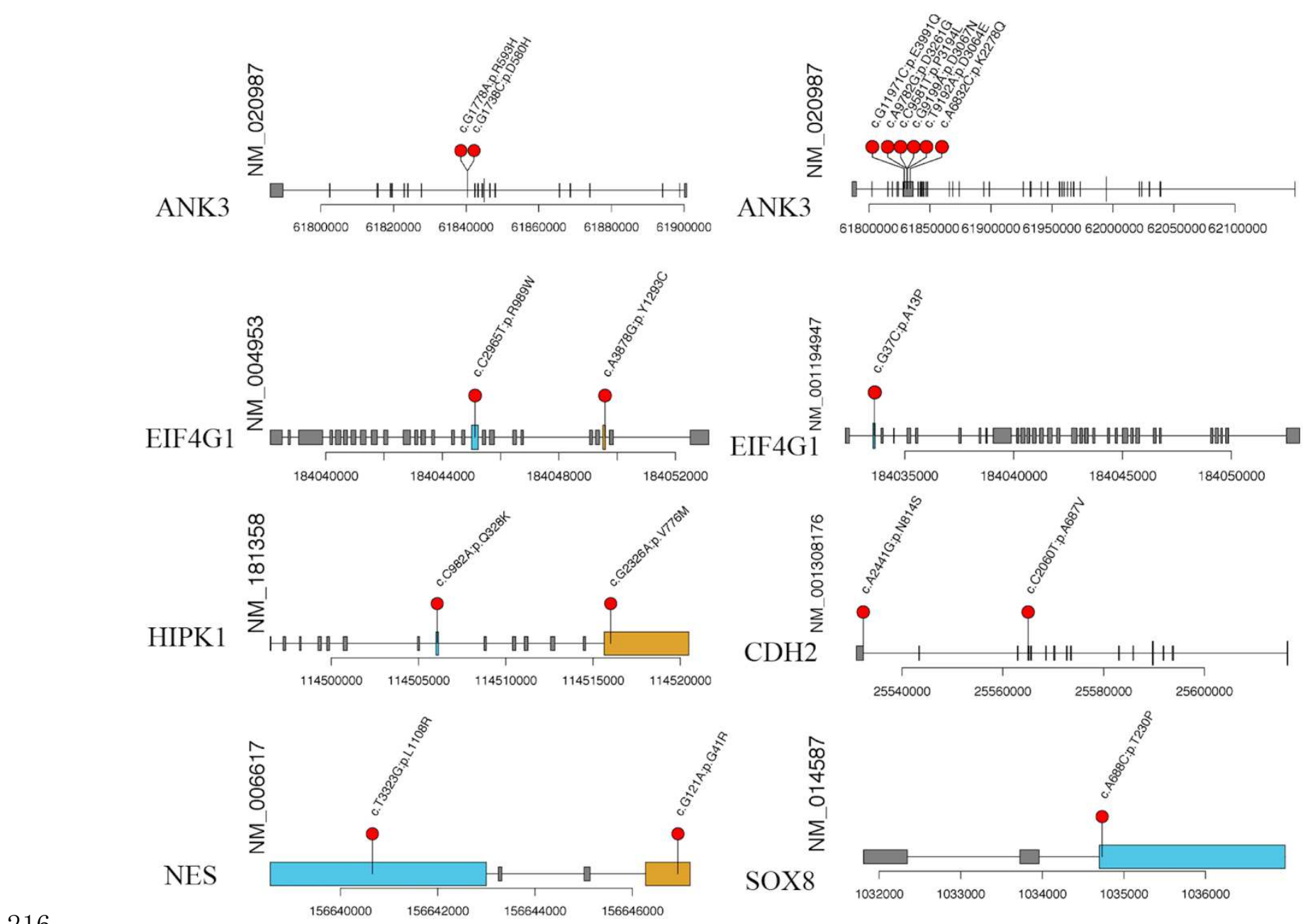

Figure 10 


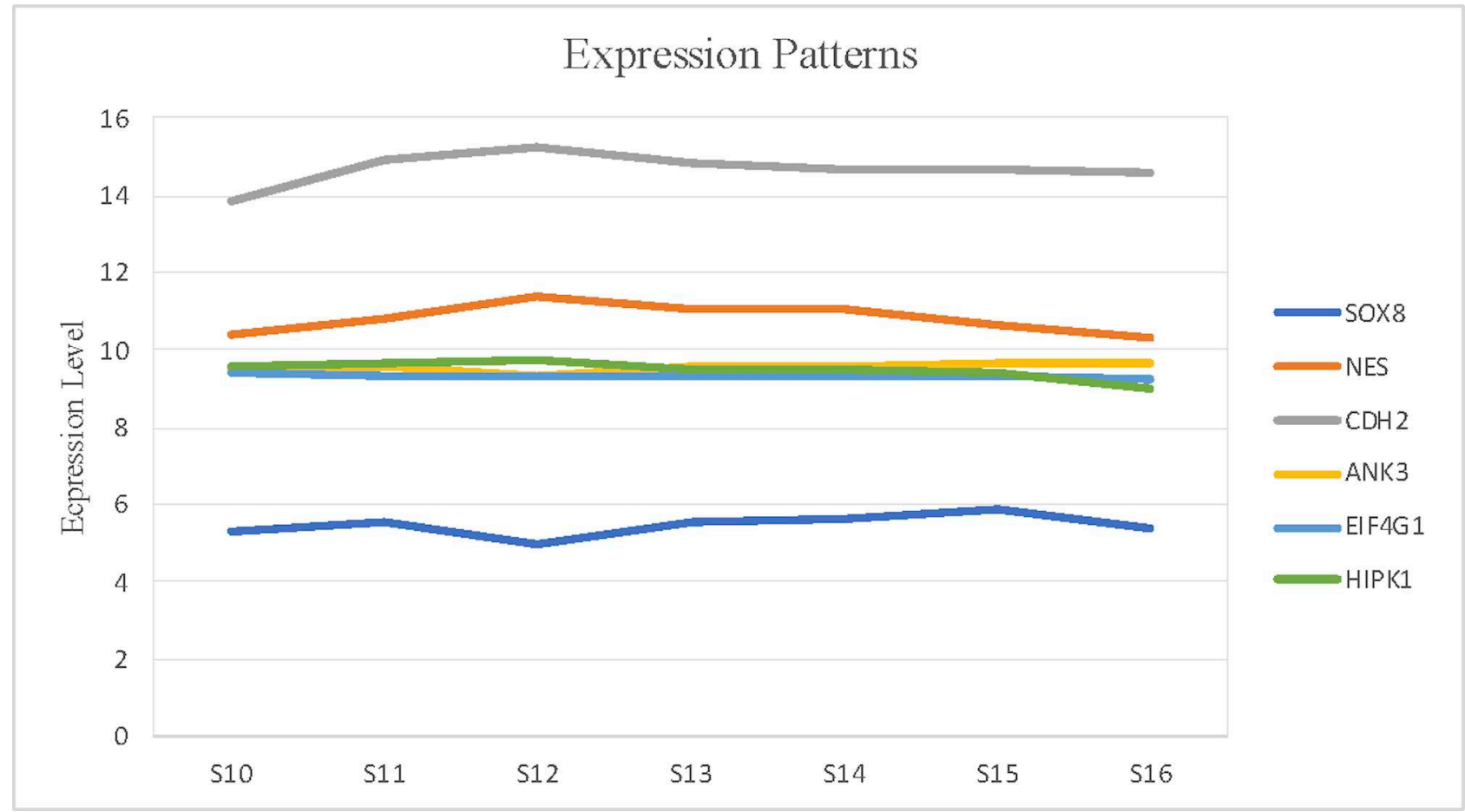

\section{Discussion}

220 As one of the most common congenital heart defects, the underlying molecular genetic mechanisms

221 of PDA are still largely unknown. In this study, we explored the clinical characteristics and performed

222 the WES to identify rare variants and candidate gene in 39 PDA patients and 100 healthy controls.

223 Through a series of bio-information filtering strategies, we prioritized the candidate genes by

224 comprehensively considering factors such Fisher exact test, mutation burden, gene network and

225 expression level. We finally identified 18 rare damaging variants in 6 totally novel candidate genes

226 (SOX8, NES, CDH2, ANK3, EIF4G1 and HIPK1) associated with PDA. In addition, CDH2 expressed the

227 most highly in human embryonic heart and seems to be the most important candidate gene in our study.

228 We hope to perform further study with larger sample size soon.

229 N-cadherin, encoded by $\mathrm{CDH} 2$, is a family of cadherins mediated cell-cell adhesion in multiple

230 tissues. Its structure consists of a single transmembrane domain, a cytoplasmic domain and five

231 conserved extracellular cadherin domains (ECI-V)[22]. In our study, we found two variants (rs25565020, 
234 showed $\mathrm{CDH} 2$ had high expressions in human embryonic hearts. Previous studies in mouse have note

235 the importance of $\mathrm{CDH} 2$ in the proper development of the heart, brain, and skeletal structures[23].

236 Interestingly, genetic analyses in zebrafish showed that mutation in in the EC-I or EC-IV domains of

237 cdh2 resulted the same heart defect phenotypes[24]. Moreover, Mayosi BM et al. used Whole exome

238 sequencing to detect novel rare variant in patients with arrhythmogenic cardiomyopathy and proved that

239 this mutation changes the conservative amino acids of the cadherin 2 protein[25]. Since the relationship

240 between $\mathrm{CDH} 2$ and PDA is unclear, additional studies are needed to determine how genetic perturbations

241 of $\mathrm{CDH} 2$ contribute to PDA.

242 In our study, 16 patients (42\%) were detected to have same variant(rs156646936) in NES. And in network

243 analysis, we observed a strong correlation between NES and known pathogenic genes. NES belongs to a

244 member of the human tissue kallikrein family of secreted serine proteases[26]. Several studies have confirmed

245 that it plays an important role in carcinogenesis, such as breast, prostate, testicular cancers and leukemia[27].

246 Further experimental evidence suggests that its function as a tumor suppressor gene may be achieved by

247 hypermethylation of the CpG island of the NES[28]. And no evidence shows mutations of NES gene in PDA.

248 ANK3 belongs to a member of the Ankyrin family that is expressed in several different isoforms in many

249 tissues. And it plays key roles in activities such as cell motility, activation, proliferation, contact, and the

250 maintenance of specialized membrane domains. In our study, 8 patients $(10 \%)$ were detected to have

251 variants in ANK3. Previous studies have shown that ANK3 variants are associated with schizophrenia, autism,

252 epilepsy and intellectual disability[29, 30]. Studies from knockout mouse models have revealed that loss of 
254 NES and ANK3 in the pathogenesis of PDA was supported by bioinformatic analyses, our study was

255 limited by the lack of experimental evidence to validate the deleteriousness of the variants.

256 EIF4G1 encoded protein which is a component of the multi-subunit protein complex EIF4F. IF4G

257 plays a crucial role in translation initiation, serving as a scaffolding protein that binds several initiation

258 factors (the cap-binding protein eIF4E, the RNA helicase eIF4A, and eIF3)[32]. In our study, 15 patients

259 were detected to have 3 types variants and the same variant(rs184033621) were detected in 14 cases.

260 EIF4G1 modulates the proliferation, apoptosis, angiogenesis of most tumour types by limiting step

261 during the initiation phase of protein synthesis and interacting with Ubiquitin-specific protease 10

262 (USP10)[33]. Moreover, phosphorylation of EIF4G1 specifically activates PKC-Ras-ERK signaling

263 pathway, which is involved in the control of growth and proliferation[34]. Disease associated with

264 EIF4G1 include Parkinson Disease, non-small cell lung carcinoma, prostate[33]. Although the

265 relationship between EIF4G1 cardiovascular development still unknow, EIF4G1 might be a potentially

266 pathogenic to PDA.

267 HIPK1 belongs to the Ser/Thr family of protein kinases and HIPK subfamily. Among its related

268 pathways are Regulation of TP53 Activity and Cardiac conduction. Homeodomain interacting protein

269 kinases, HIPK1 and HIPK2, play a key role in embryonic development by regulating TGF- $\beta$-dependent

270 angiogenesis[35, 36]. HIPK1 loss-of-function conditional knockout mice exhibit defects in

271 primitive/definitive hematopoiesis, vasculogenesis, angiogenesis and neural tube closure[36]. In addition,

272 HIPK1 can interact with homeobox proteins and other transcription factors to regulate a variety of

273 biological processes, such as signal transduction, apoptosis, embryonic development, retinal vascular 
283 Sox 8 might associated with hypoxia-induced cell injury by activating the PI3K/AKT/mTOR pathway

284 and MAPK[39]. Interestingly, the closure of DA after birth is closely related to blood oxygenation level

285 and hypoxia can lead to the increase of endogenous PGE2 release, and directly lead to the opening of the

286 ductus arteriosus[1]. Thus far, SOX8 may be a novel candidate gene in the pathogenesis.

\section{Conclusions}

\section{Abbreviations}


WES: Whole-exome Sequencing

VSMCs: Vascular smooth muscle cells

DA: Ductus arteriosus

PPI: Protein-protein interaction

GO: Gene Ontology

\section{References}

304 1. Benitz WE, Committee on F, Newborn AAoP. Patent Ductus Arteriosus in Preterm Infants. Pediatrics. 2016; 137.

2. Hoffman Jl, Kaplan S. The incidence of congenital heart disease. J Am Coll Cardiol. 2002; 39:1890-900.

308 3. Mitra S, Florez ID, Tamayo ME, Mbuagbaw L, Vanniyasingam T, Veroniki AA et al. Association of Placebo, Indomethacin, Ibuprofen, and Acetaminophen With Closure of Hemodynamically Significant Patent Ductus Arteriosus in Preterm Infants: A Systematic Review and Meta-analysis. JAMA. 2018; 319:1221-38.

312 4. Crockett SL, Berger CD, Shelton EL, Reese J. Molecular and mechanical factors 
315 5. Li N, Subrahmanyan L, Smith E, Yu X, Zaidi S, Choi M et al. Mutations in the Histone

316 Modifier PRDM6 Are Associated with Isolated Nonsyndromic Patent Ductus Arteriosus.

317 Am J Hum Genet. 2016; 98:1082-91.

318 6. Gravholt CH, Viuff MH, Brun S, Stochholm K, Andersen NH. Turner syndrome:

319 mechanisms and management. Nat Rev Endocrinol. 2019; 15:601-14.

320 7. Yang D, Liu BC, Luo J, Huang TX, Liu CT. Kartagener syndrome. QJM. 2019; 112:297-8.

321 8. Groth KA, Skakkebaek A, Host C, Gravholt CH, Bojesen A. Clinical review: Klinefelter

322 syndrome--a clinical update. J Clin Endocrinol Metab. 2013; 98:20-30.

323 9. Satoda M, Zhao F, Diaz GA, Burn J, Goodship J, Davidson HR et al. Mutations in TFAP2B

324 cause Char syndrome, a familial form of patent ductus arteriosus. Nat Genet. 2000;

$325 \quad 25: 42-6$.

326 10. Vanlerberghe C, Jourdain AS, Ghoumid J, Frenois F, Mezel A, Vaksmann G et al. Holt-

327 Oram syndrome: clinical and molecular description of 78 patients with TBX5 variants. Eur

$328 \quad$ J Hum Genet. 2019; 27:360-8.

329 11. Pannone L, Bocchinfuso G, Flex E, Rossi C, Baldassarre G, Lissewski C et al. Structural,

330 Functional, and Clinical Characterization of a Novel PTPN11 Mutation Cluster Underlying

$331 \quad$ Noonan Syndrome. Hum Mutat. 2017; 38:451-9.

332 12. Harakalova M, van der Smagt J, de Kovel CG, Van't Slot R, Poot M, Nijman IJ et al.

333 Incomplete segregation of MYH11 variants with thoracic aortic aneurysms and

334 dissections and patent ductus arteriosus. Eur J Hum Genet. 2013; 21:487-93. 
335 13. Erdogan F, Larsen LA, Zhang L, Tumer Z, Tommerup N, Chen W et al. High frequency of 336 submicroscopic genomic aberrations detected by tiling path array comparative genome hybridisation in patients with isolated congenital heart disease. J Med Genet. 2008; 45:704-9.

14. Li H, Durbin R. Fast and accurate short read alignment with Burrows-Wheeler transform. Bioinformatics. 2009; 25:1754-60.

341 15. Okonechnikov K, Conesa A, Garcia-Alcalde F. Qualimap 2: advanced multi-sample quality control for high-throughput sequencing data. Bioinformatics. 2016; 32:292-4.

343 16. Gezsi A, Bolgar B, Marx P, Sarkozy P, Szalai C, Antal P. VariantMetaCaller: automated fusion of variant calling pipelines for quantitative, precision-based filtering. BMC Genomics. 2015; 16:875.

17. Richards S, Aziz N, Bale S, Bick D, Das S, Gastier-Foster J et al. Standards and guidelines Pathology. Genet Med. 2015; 17:405-24.

350 18. Gene Ontology C. Gene Ontology Consortium: going forward. Nucleic Acids Res. 2015; 43:D1049-56.

19. Kanehisa M, Furumichi M, Tanabe M, Sato Y, Morishima K. KEGG: new perspectives on genomes, pathways, diseases and drugs. Nucleic Acids Res. 2017; 45:D353-D61.

354 20. Brohee S, Faust K, Lima-Mendez G, Vanderstocken G, van Helden J. Network Analysis 
21. O'Rahilly R. Human embryo. Nature. 1987; 329:385.

22. Alimperti S, Andreadis ST. $\mathrm{CDH} 2$ and $\mathrm{CDH} 11$ act as regulators of stem cell fate decisions. Stem Cell Res. 2015; 14:270-82.

23. Radice GL, Rayburn H, Matsunami H, Knudsen KA, Takeichi M, Hynes RO.

Developmental defects in mouse embryos lacking N-cadherin. Dev Biol. 1997; 181:64-

78.

24. Masai I, Lele Z, Yamaguchi M, Komori A, Nakata A, Nishiwaki Y et al. N-cadherin mediates retinal lamination, maintenance of forebrain compartments and patterning of retinal neurites. Development. 2003; 130:2479-94.

25. Mayosi BM, Fish M, Shaboodien G, Mastantuono E, Kraus S, Wieland T et al. Identification of Cadherin $2(\mathrm{CDH} 2)$ Mutations in Arrhythmogenic Right Ventricular Cardiomyopathy. Circ Cardiovasc Genet. 2017; 10.

26. Luo L, Herbrick JA, Scherer SW, Beatty B, Squire J, Diamandis EP. Structural characterization and mapping of the normal epithelial cell-specific 1 gene. Biochem Biophys Res Commun. 1998; 247:580-6.

27. Luo LY, Rajpert-De Meyts ER, Jung K, Diamandis EP. Expression of the normal epithelial cell-specific 1 (NES1; KLK10) candidate tumour suppressor gene in normal and malignant testicular tissue. $\mathrm{Br}$ J Cancer. 2001; 85:220-4.

28. Li B, Goyal J, Dhar S, Dimri G, Evron E, Sukumar S et al. CpG methylation as a basis for breast tumor-specific loss of NES1/kallikrein 10 expression. Cancer Res. 2001; 61:801421. 
29. Leussis MP, Berry-Scott EM, Saito M, Jhuang H, de Haan G, Alkan O et al. The ANK3 bipolar disorder gene regulates psychiatric-related behaviors that are modulated by lithium and stress. Biol Psychiatry. 2013; 73:683-90.

30. Wirgenes KV, Tesli M, Inderhaug E, Athanasiu L, Agartz I, Melle I et al. ANK3 gene expression in bipolar disorder and schizophrenia. Br J Psychiatry. 2014; 205:244-5. arrhythmia syndrome caused by loss of ankyrin-B function. Proc Natl Acad Sci U S A. $2004 ; 101: 9137-42$.

32. Haimov O, Sehrawat U, Tamarkin-Ben Harush A, Bahat A, Uzonyi A, Will A et al. Dynamic Interaction of Eukaryotic Initiation Factor 4G1 (elF4G1) with elF4E and elF1 Underlies Scanning-Dependent and -Independent Translation. Mol Cell Biol. 2018; 38. initiation factor 4 gamma 1 (EIF4G1) in NSCLC. Oncotarget. 2016; 7:24242-51.

390 34. Dobrikov M, Dobrikova E, Shveygert M, Gromeier M. Phosphorylation of eukaryotic binding to Mnk1. Mol Cell Biol. 2011; 31:2947-59.

393 35. Aikawa Y, Nguyen LA, Isono K, Takakura N, Tagata Y, Schmitz ML et al. Roles of HIPK1 and HIPK2 in AML1- and p300-dependent transcription, hematopoiesis and blood vessel formation. EMBO J. 2006; 25:3955-65. 
36. Shang Y, Doan CN, Arnold TD, Lee S, Tang AA, Reichardt LF et al. Transcriptional corepressors HIPK1 and HIPK2 control angiogenesis via TGF-beta-TAK1-dependent mechanism. PLoS Biol. 2013; 11:e1001527.

37. Haseeb A, Lefebvre V. The SOXE transcription factors-SOX8, SOX9 and SOX10-share a bi-partite transactivation mechanism. Nucleic Acids Res. 2019; 47:6917-31. Expression of Sox8, Sox9 and Sox10 in the developing valves and autonomic nerves of the embryonic heart. Mech Dev. 2002; 118:199-202. Protects H9c2 Cells against Hypoxia-Induced Injury by Targeting MicroRNA-139. Cell Physiol Biochem. 2017; 44:857-69.

\section{Figure legends}

408 Figure 1: Bioinformatics filtering strategy workflow for the candidate genes. Through a series of

409 filtering methods, we finally identified 6 candidate genes. The potentially damaging variants in candidate 410 genes were subjected to validation via human embryonic heart expression analysis.

411 Figure 2: The comparisons of the rare damaging variants between the PDA and control groups.

412 The number of variants in each variant classification and SNV class between cases and controls are

413 presented in (A), (B), (C) and (D), respectively.

414 Figure 3: Single SNP allele frequency and genotype frequency p-values were obtained using the

415 fisher exact test. X-axis represents the position of each snp (represented in circles) on human 

represented in the figure.

418 Figure 4: Heatmap representing the top 10 genes identified in Burden analysis. Heatmap that shows

419 the mutational burden (P-value $<0.05)$ of the top ten gene based on gene-based burden analysis in PDA

420 patients. The heatmap was generated by using R package, the mutation values were normalized per gene

421 over all PDA samples. Each box in the heatmap represent a single variant in a case, with the dark red

422 indicating high gene mutation ration in gene-based Burden analysis.

423 Figure 5: Bubble plot of the GO analysis. Bubble plot summarizing enrichment for the most

424 significant biological process GO terms associated to differentially expressed genes. The bubble size 425 indicates the frequency of the GO term, while the color indicates the P-value.

426 Figure 6: Bubble plot of the KEGG pathway analysis. The representative enriched pathways shown 427 by KEGG analysis. The bubble size indicates the frequency of the KEGG term, while the color indicates 428 the P-value.

429 Figure 7: Interaction between our candidate genes and known CHD-related genes. PPI network was

430 generated by Cytoscape software and our candidate pathogenic genes and the known CHD-related genes were

431 uploaded in STRING database. Each node represents one gene, and each edge represents the protein-

432 protein interaction collected from BioGRID.

433 Figure 8: Interaction between our candidate genes and known PDA-related genes. PPI network was

434 generated by Cytoscape software and Our candidate pathogenic genes and the known CHD-related genes

435 were uploaded in STRING database. Each node represents one gene, and each edge represents the protein-

436 protein interaction collected from BioGRID. 
438 location of rare variant on the encoded proteins or protein domains.

439 Figure 10: Expression of candidate genes in human embryonic heart. The expression patterns of

440 candidate genes in human embryonic heart at different stages of S10 to S16 were analyzed by microarray.

$441 \mathrm{X}$-axis represents the different stages of human embryonic heart, while the Y-axis indicates the level of 442 gene expression.

\section{Declarations}

\section{Ethics approval and consent to participate}

445 The studies involving human participants were reviewed and approved by the Medical Ethics Committee 446 of Xinhua Hospital (Approval No. XHEC-D-2020-001). Written informed consent to participate in this 447 study was provided by the participants' legal guardian/next of kin.

\section{Consent for publication}

449 Not applicable.

\section{$450 \quad$ Availability of data and materials}

451 The datasets supporting the conclusions of this article are available in the NCBI SRA repository and have

452 been compiled into the following repository for ease of access:

453 https://www.ncbi.nlm.nih.gov/sra/?term=SRP288538. The accession number is SRP288538.

\section{Competing interests}

455 The authors declare that they have no competing interests.

\section{Author information}




\section{Corresponding author}

464 Correspondence to Bo Chen.

\section{$465 \quad$ Funding}

466 This study received financial supports from National Natural Science Foundation of China (82070386), the

467 Project of Shanghai Municipal Health Commission (Grant No. 201940393).

\section{Authors' contributions}

PZ and BC contributed to design of the study and performed the statistical analysis. HY, QZ, and BY manuscript revision, read and approved the submitted version.

474 Not applicable.

\section{Supplementary information}


478 are related to cardiac and vascular development and PDA. 


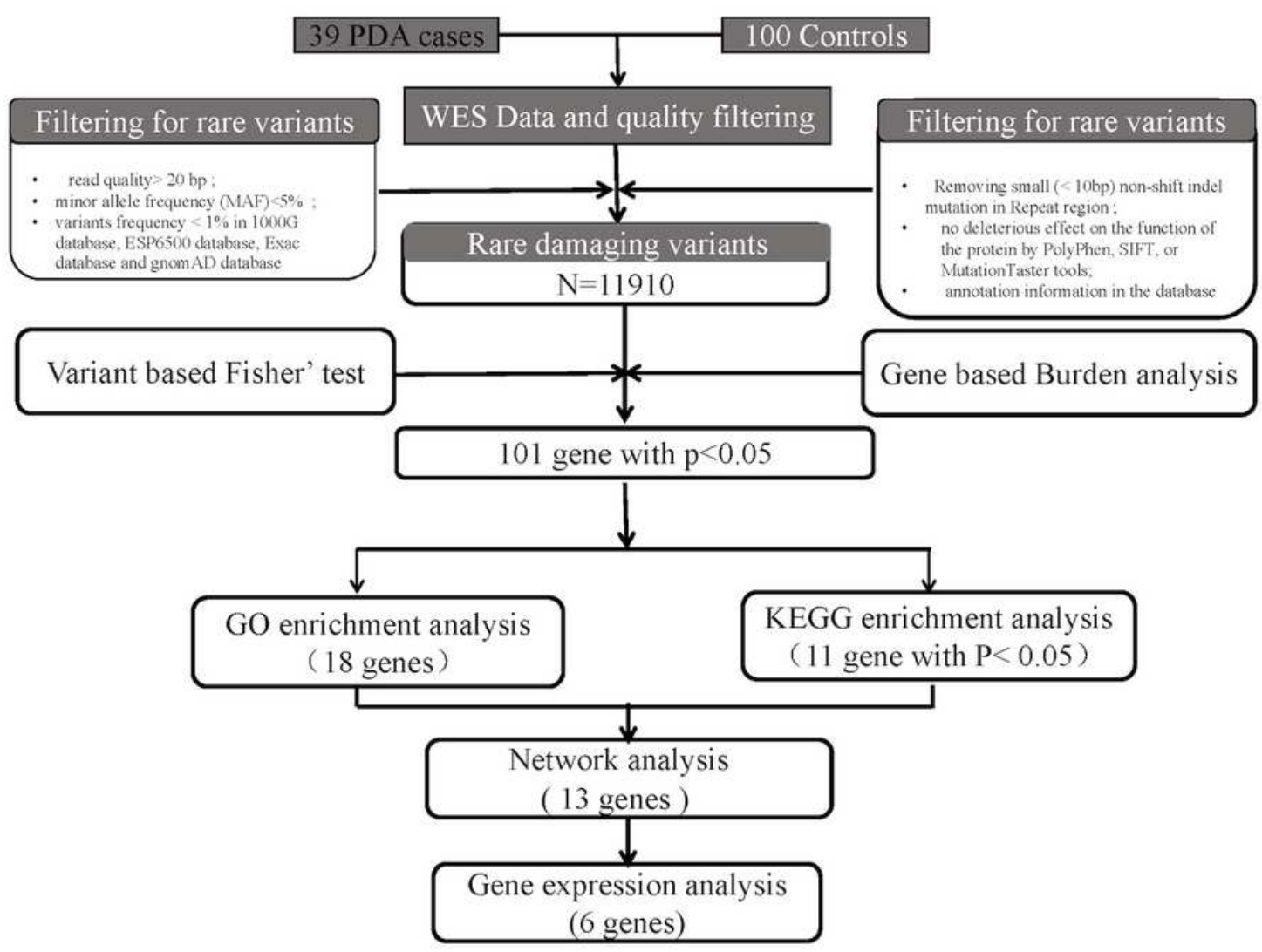

\section{Figure 1}

Bioinformatics filtering strategy workflow for the candidate genes. Through a series of filtering methods, we finally identified 6 candidate genes. The potentially damaging variants in candidate genes were subjected to validation via human embryonic heart expression analysis. 
A

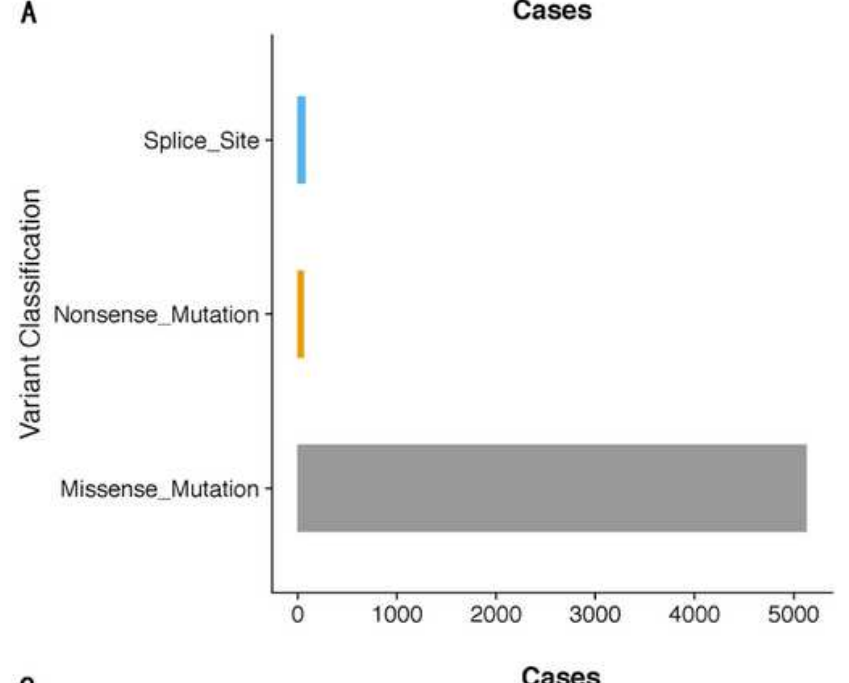

C

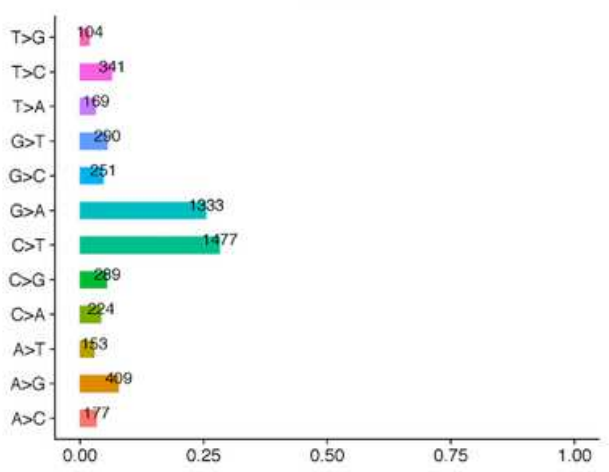

B

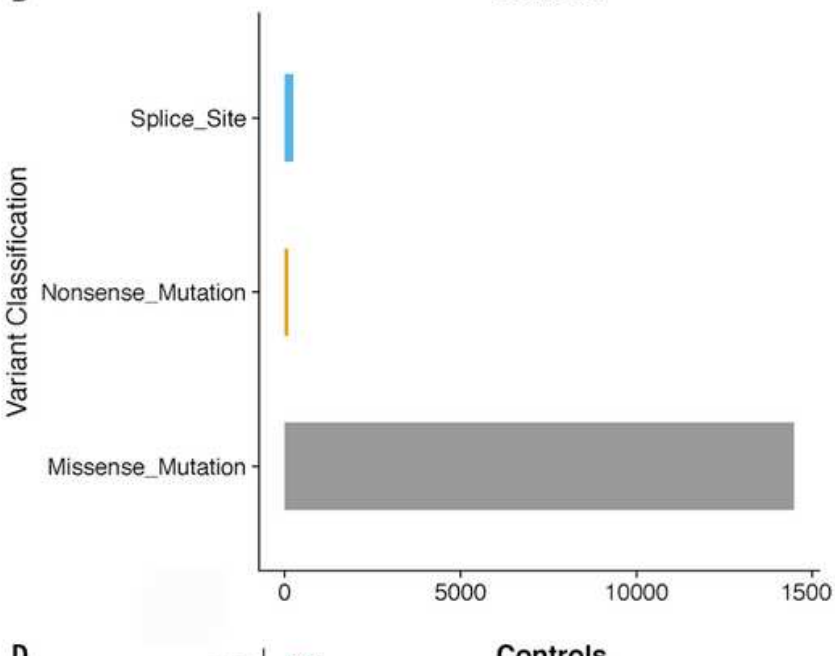

D

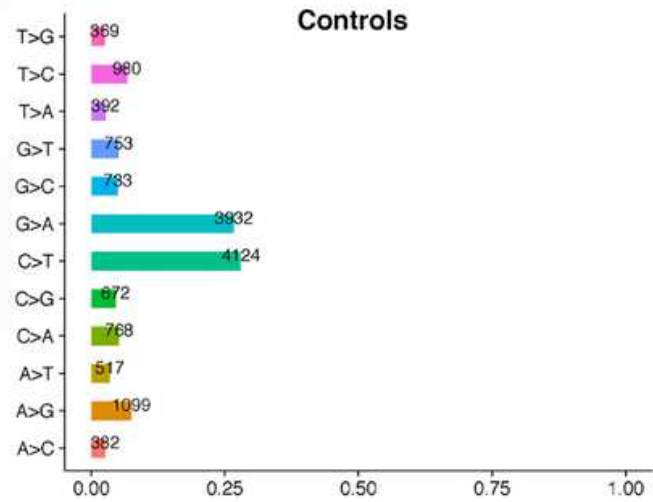

\section{Figure 2}

The comparisons of the rare damaging variants between the PDA and control groups. The number of variants in each variant classification and SNV class between cases and controls are presented in (A), (B), (C) and (D), respectively. 


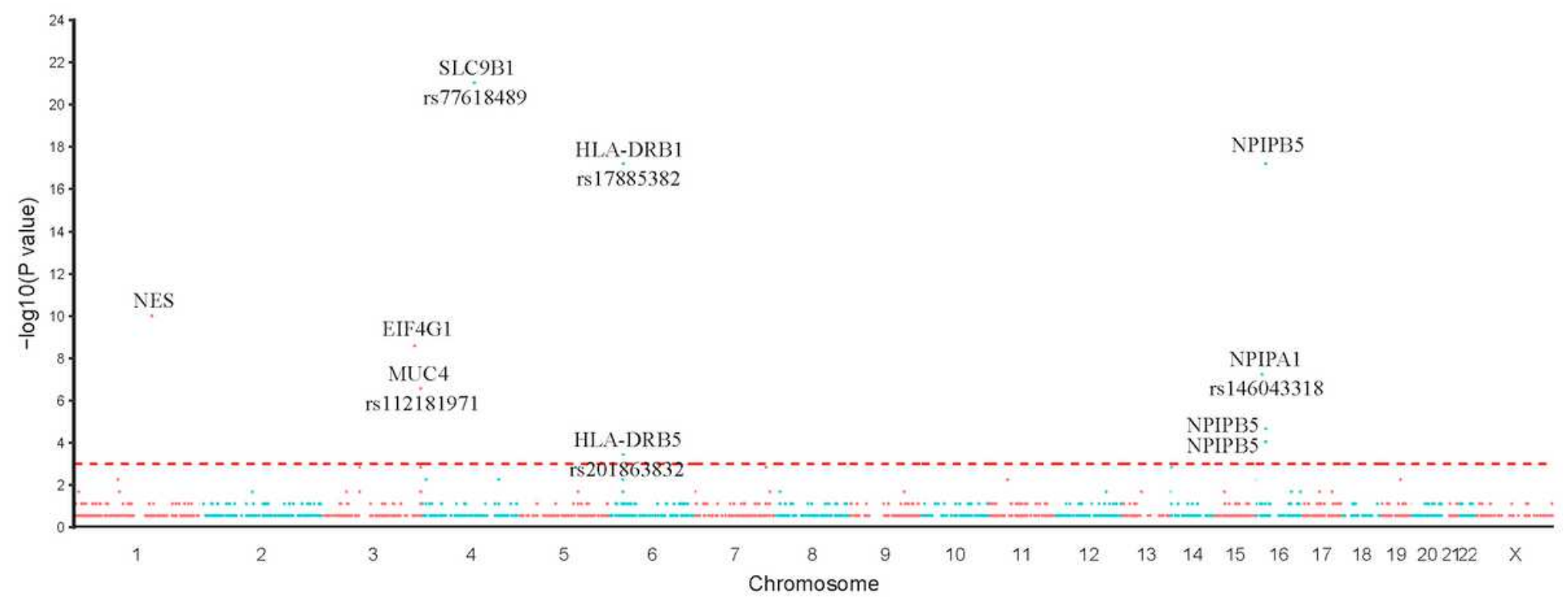

\section{Figure 3}

Single SNP allele frequency and genotype frequency $\mathrm{p}$-values were obtained using the fisher exact test. Xaxis represents the position of each snp (represented in circles) on human chromosome, Y-axis is the log P-value of Fisher Exact test. Top ten variants in our study were represented in the figure.

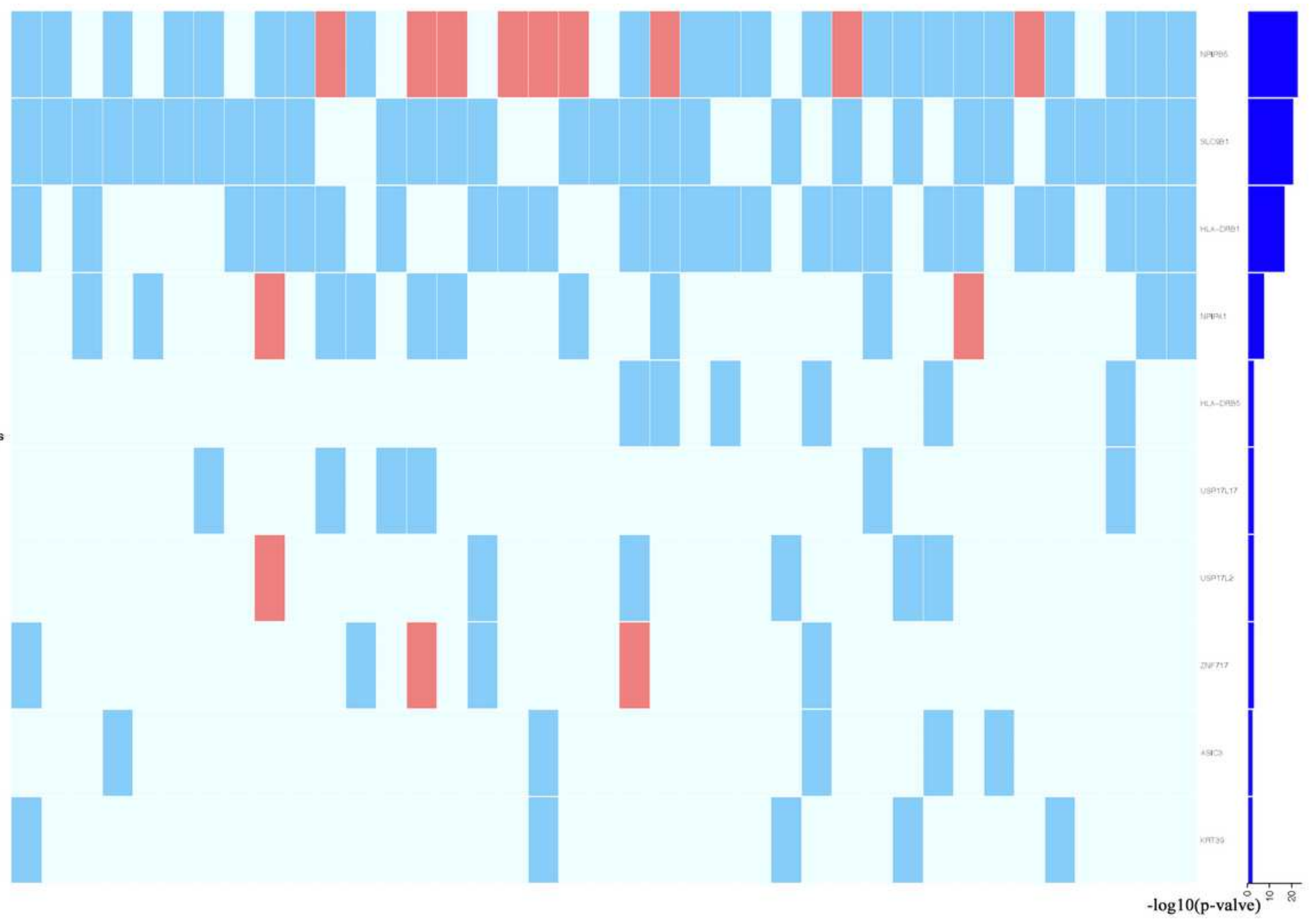




\section{Figure 4}

Heatmap representing the top 10 genes identified in Burden analysis. Heatmap that shows the mutational burden (P-value $<0.05$ ) of the top ten gene based on gene-based burden analysis in PDA patients. The heatmap was generated by using $\mathrm{R}$ package, the mutation values were normalized per gene over all PDA samples. Each box in the heatmap represent a single variant in a case, with the dark red indicating high gene mutation ration in gene-based Burden analysis.

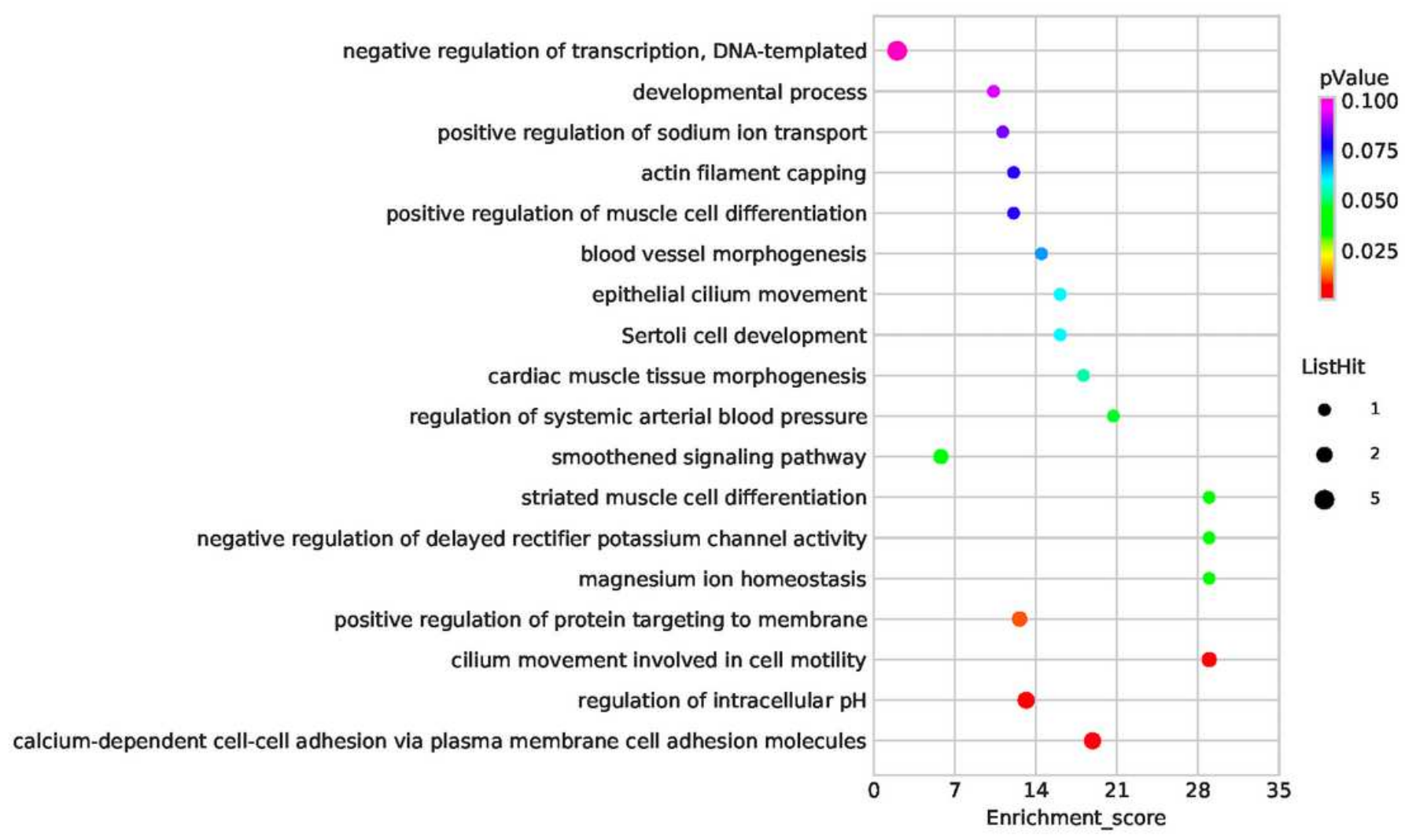

\section{Figure 5}

Bubble plot of the $\mathrm{GO}$ analysis. Bubble plot summarizing enrichment for the most significant biological process GO terms associated to differentially expressed genes. The bubble size indicates the frequency of the GO term, while the color indicates the P-value. 


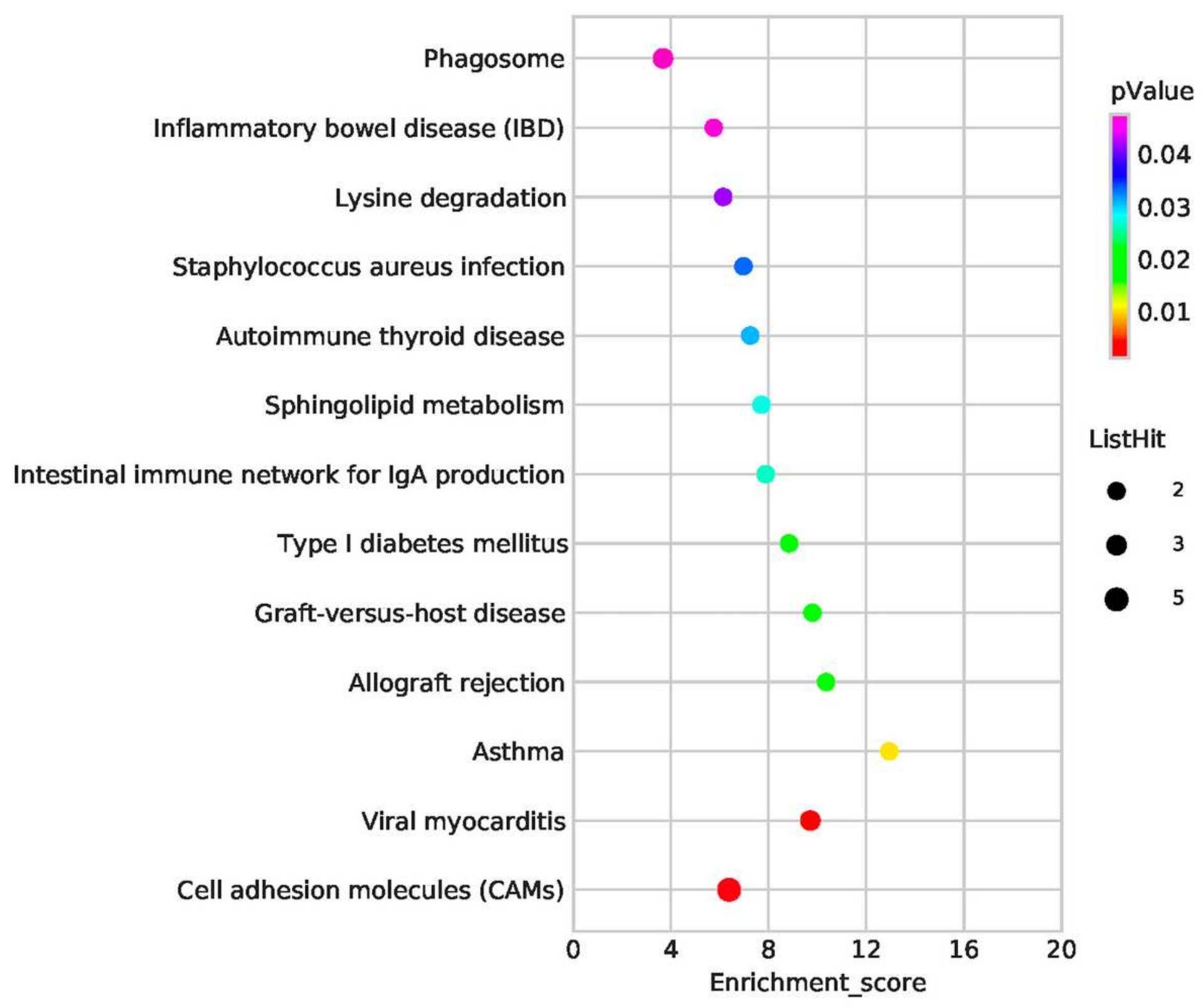

Figure 6

Bubble plot of the KEGG pathway analysis. The representative enriched pathways shown by KEGG analysis. The bubble size indicates the frequency of the KEGG term, while the color indicates the P-value. 


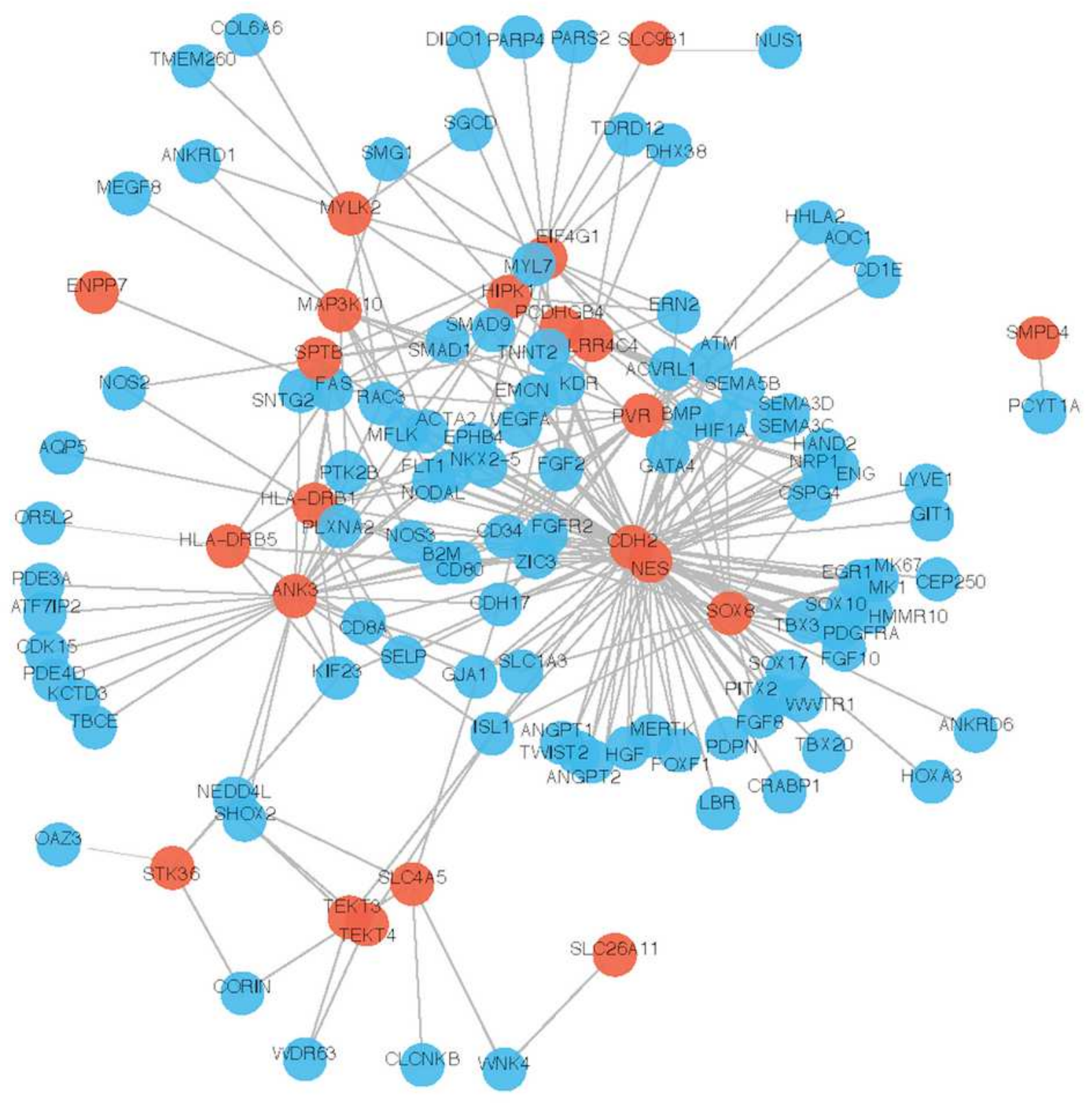

Figure 7

Interaction between our candidate genes and known CHD-related genes. PPI network was generated by Cytoscape software and our candidate pathogenic genes and the known CHD-related genes were uploaded in STRING database. Each node represents one gene, and each edge represents the proteinprotein interaction collected from BioGRID. 


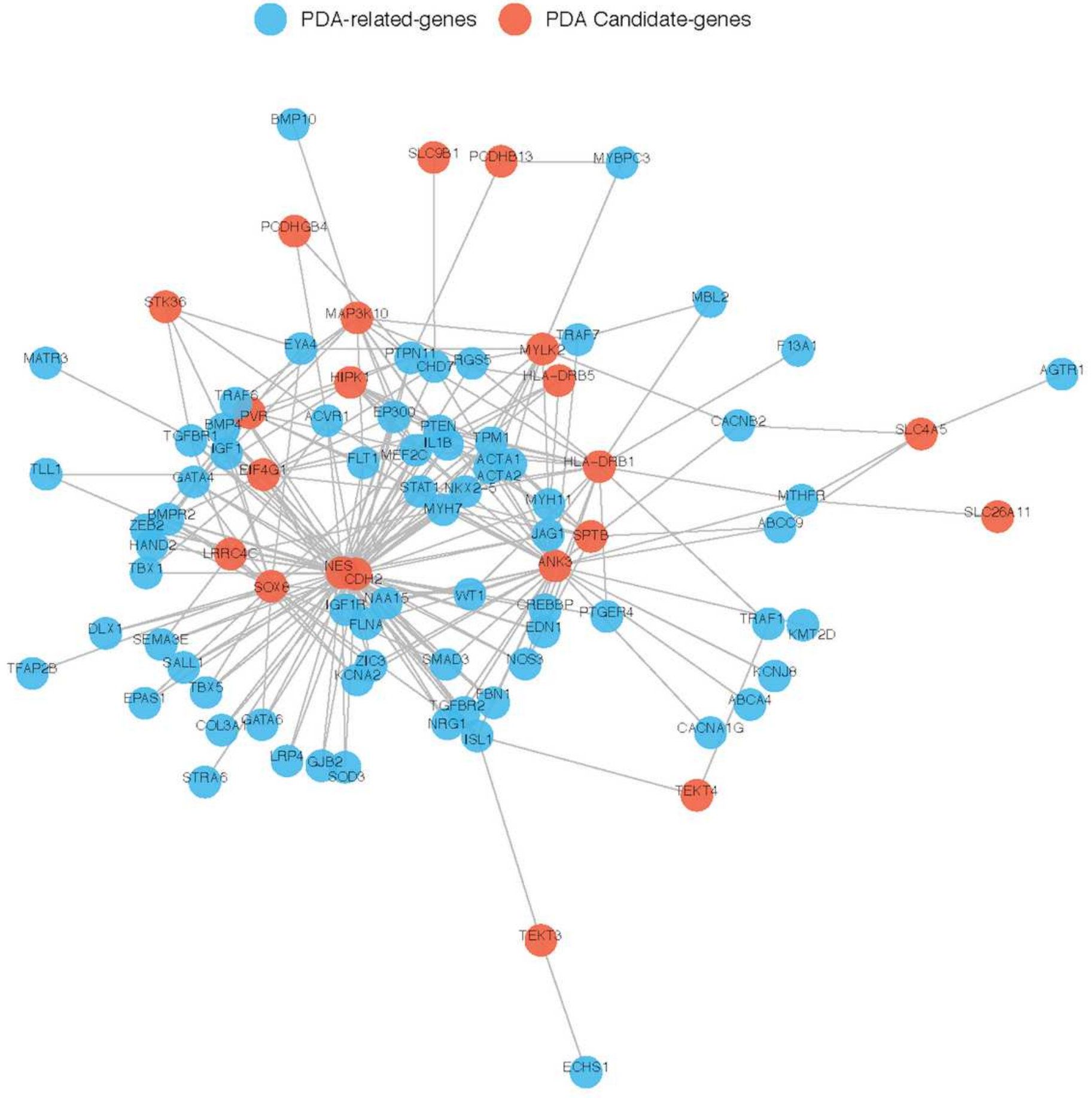

\section{Figure 8}

Interaction between our candidate genes and known PDA-related genes. PPI network was generated by Cytoscape software and Our candidate pathogenic genes and the known CHD-related genes were uploaded in STRING database. Each node represents one gene, and each edge represents the proteinprotein interaction collected from BioGRID. 

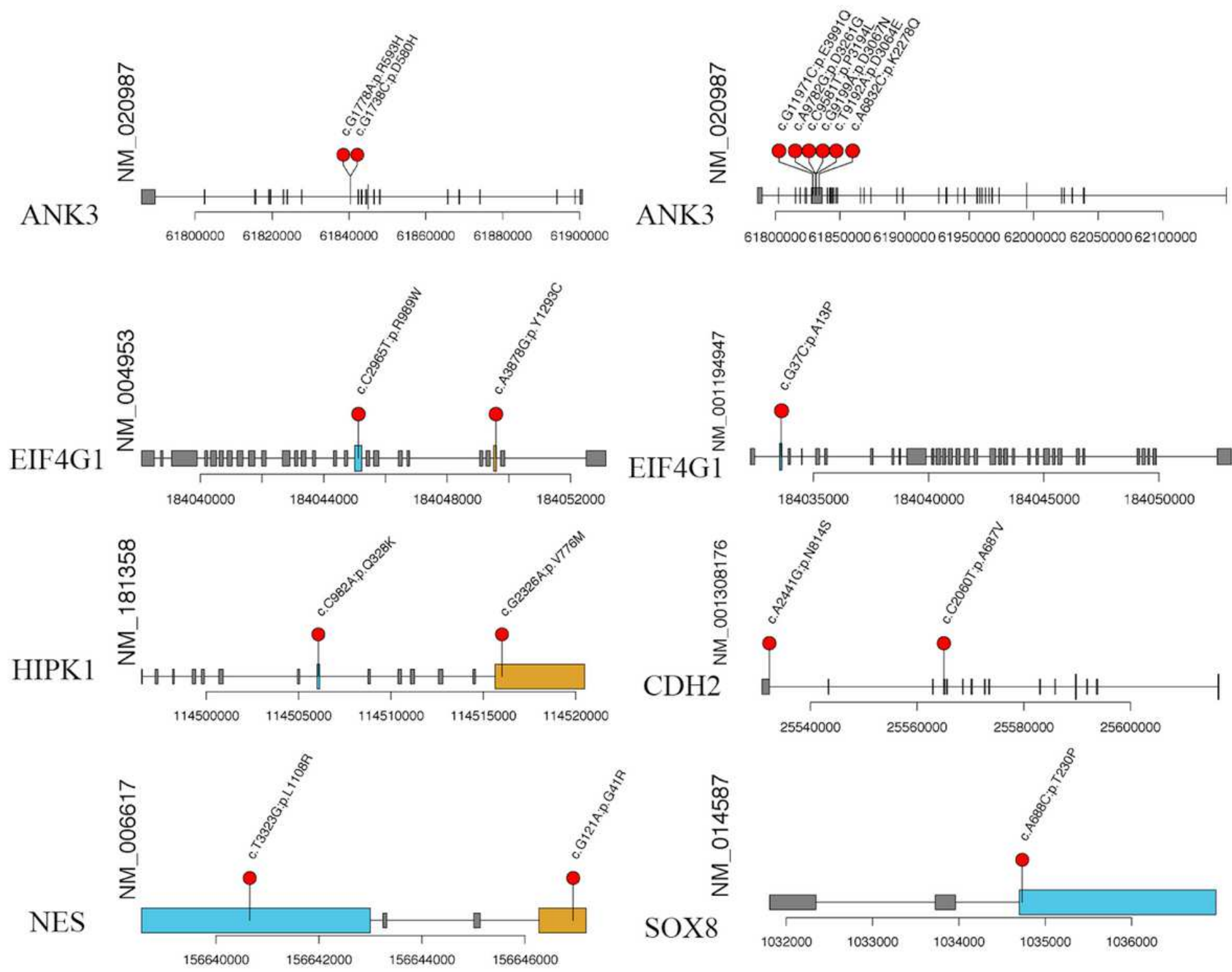

\section{Figure 9}

The specific amino acid sites of variants of our candidate gene. The red balls represent the location of rare variant on the encoded proteins or protein domains. 


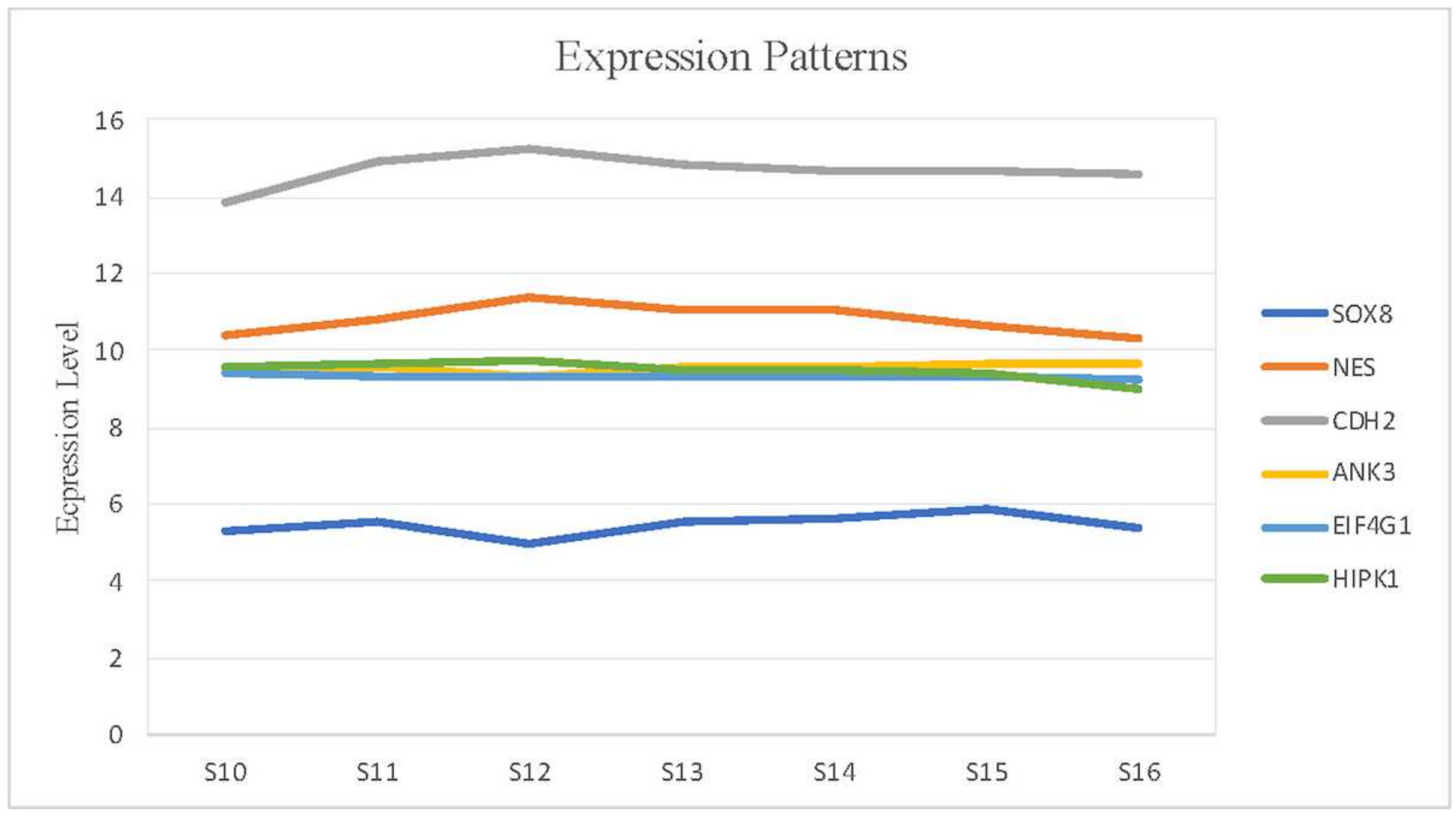

Figure 10

Expression of candidate genes in human embryonic heart. The expression patterns of candidate genes in human embryonic heart at different stages of $S 10$ to $S 16$ were analyzed by microarray. $X$-axis represents the different stages of human embryonic heart, while the $Y$-axis indicates the level of gene expression.

\section{Supplementary Files}

This is a list of supplementary files associated with this preprint. Click to download.

- supplementaryfile.docx 